\title{
Model-independent determination of the triple Higgs coupling at $e^{+} e^{-}$colliders
}

\author{
Tim Barklow, ${ }^{1}$ Keisuke Fujii, ${ }^{2}$ Sunghoon Jung, ${ }^{1,3}$ Michael E. Peskin, ${ }^{1}$ and Junping Tian $^{4}$ \\ ${ }^{1}$ SLAC, Stanford University, Menlo Park, California 94025, USA \\ ${ }^{2}$ High Energy Accelerator Research Organization (KEK), Tsukuba, Ibaraki 305-0801, Japan \\ ${ }^{3}$ Department of Physics and Astronomy, Seoul National University, Seoul 08826, Korea \\ ${ }^{4}$ ICEPP, University of Tokyo, Hongo, Bunkyo-ku, Tokyo 113-0033, Japan
}

(Received 6 September 2017; published 20 March 2018)

\begin{abstract}
The observation of Higgs pair production at high-energy colliders can give evidence for the presence of a triple Higgs coupling. However, the actual determination of the value of this coupling is more difficult. In the context of general models for new physics, double Higgs production processes can receive contributions from many possible beyond-Standard-Model effects. This dependence must be understood if one is to make a definite statement about the deviation of the Higgs field potential from the Standard Model. In this paper, we study the extraction of the triple Higgs coupling from the process $e^{+} e^{-} \rightarrow Z h h$. We show that, by combining the measurement of this process with other measurements available at a $500 \mathrm{GeV} e^{+} e^{-}$collider, it is possible to quote model-independent limits on the effective field theory parameter $c_{6}$ that parametrizes modifications of the Higgs potential. We present precise error estimates based on the anticipated International Linear Collider physics program, studied with full simulation. Our analysis also gives new insight into the model-independent extraction of the Higgs boson coupling constants and total width from $e^{+} e^{-}$data.
\end{abstract}

DOI: 10.1103/PhysRevD.97.053004

\section{INTRODUCTION}

The discovery of the Higgs boson in 2012 [1,2] closed one chapter in our understanding of elementary particle physics but opened another. The observation of this particle, at a relatively low mass and with large couplings to $W, Z$, and heavy fermions, confirmed the picture of the Standard Model that masses originate in the vacuum expectation value of a scalar field. At the same time, this observation deepened the mysteries associated with this particle, and also offered a path to solving these mysteries through precision measurements of the Higgs boson's properties.

The goal of this paper is to develop methods for the precision extraction of Higgs boson couplings using effective field theory to represent the most general effects of new physics on the Higgs boson. Effective field theory (EFT) has been applied to the theory of a Higgs boson in many papers, for example, Refs. [3-5] and has been adopted as a canonical framework for analyzing Higgs boson measurements at the LHC [6]. Still, we feel that the

Published by the American Physical Society under the terms of the Creative Commons Attribution 4.0 International license. Further distribution of this work must maintain attribution to the author(s) and the published article's title, journal citation, and DOI. Funded by SCOAP ${ }^{3}$. full power of this formalism is not appreciated. The reason for this is that a fully general treatment of EFT brings in a very large number of free parameters. It has not been clear how to constrain all of these parameters simultaneously from experimental measurements [7].

In this paper, we will show that this problem can be solved by making use of the large number of observables that can be measured with high precision at future $e^{+} e^{-}$ colliders. In our analysis, we analyze the extension of the Standard Model (SM) by the addition of ten effective operators that describe the most general new physics effects on the couplings of the Higgs boson to the $W, Z$, and $\gamma$ and the light leptons. We show how to determine the coefficients of these operators systematically. We apply this method to solve an important problem involving the measurement of the Higgs boson self-coupling. We also present formulas that extend this method to a general, model-independent approach to the extraction of Higgs boson couplings from data.

The specific aim of this paper is to solve the following problem for the Higgs boson self-coupling: the Higgs boson self-coupling is predicted by the SM. The experimental test of this prediction is of great importance both for our basic understanding of electroweak symmetry breaking and for the linkage of this issue to other questions such as Higgs $C P$ violation and electroweak baryogenesis [8]. If the SM were exact except for a perturbation that 
changes the triple Higgs coupling, it would be possible to measure this coupling by observing a change in the rate of double Higgs production. This measurement has been studied in some detail for $g g \rightarrow h h$ at hadron colliders [9-11], for $e^{+} e^{-} \rightarrow Z h h$ [12] and for the vector-boson fusion processes $e^{+} e^{-} \rightarrow \nu \bar{\nu} h h[12,13]$, and $u d \rightarrow d u h h$ [11]. In some cases, future experiments would be highly sensitive to a deviation of the rate from the SM prediction. A series of papers, beginning with Ref. [14], have even discussed extracting the triple Higgs coupling from single Higgs production measurements, through its effects in loop diagrams. However, the assumption that the triple Higgs coupling is altered by some effect of new physics while all other Higgs boson couplings remained unchanged is extremely artificial. It is more likely that new physics alters many of the couplings of the Higgs boson and alters the rate of single and double Higgs production through many different vertices. But how, then, can we distinguish the effects of changes in the Higgs boson potential from perturbations induced by other new physics effects?

This question has hardly been studied in the literature, and its resolution is not straightforward. Reference [15] studied the influence of a second operator perturbation of the SM and showed that this effect can be distinguished from a change in the triple Higgs coupling by studying the dependence of the double Higgs production cross section on $m(h h)$ at center-of-mass energies well above threshold. References [16-18] studied the process $g g \rightarrow h h$ at proton colliders and suggested measurements beyond the total cross section measurements that discriminate contributions of different operators. Reference [19] studied the discrimination of loop effects of the triple Higgs coupling in single Higgs processes from other EFT effects. In all of these cases, the extension of the method to high precision and to general new physics perturbations seems very challenging.

The best way to attack this problem is to enumerate all possible new physics effects that influence the cross section for double Higgs production and to constrain them one by one in a systematic way, leaving, at the end, only the triple Higgs coupling as a free parameter. In this paper, we explain how to do that through the use of the EFT parametrization of possible deviations from the SM. We concentrate on the extraction of the triple Higgs coupling from the rate of the reaction $e^{+} e^{-} \rightarrow Z h h$, which can be measured already at a $500 \mathrm{GeV} e^{+} e^{-}$collider. Effects on the $e^{+} e^{-} \rightarrow Z h h$ cross section from dimension-six EFT operators have been studied previously in Ref. [20].

Our analysis will involve a total of 17 EFT operator coefficients. Of these, one is the parameter $c_{6}$ that shifts the triple Higgs coupling, nine others govern the couplings among vector bosons, leptons, and the Higgs boson, while another seven appear in other Higgs decay amplitudes that will enter our analysis. This seems at first sight extremely complex, but we will see that each coefficient has its place and can be constrained in a physically apparent way.
The outline of this paper is as follows. In Sec. II, we set up our formalism for the EFT analysis of Higgs and vectorboson process. We present a basic strategy for our analysis by writing the potentially measurable vector-boson, lepton, and Higgs couplings in terms of EFT coefficients. We justify the restriction to this parameter set and discuss some approximations we make to simplify the analysis. And, we present our method for including the constraints on the EFT coefficients coming from precision electroweak measurements. In Sec. III, we present the constraints from measurements of $e^{+} e^{-} \rightarrow W^{+} W^{-}$at future $e^{+} e^{-}$colliders and describe these constraints quantitatively using the results of full-simulation studies for the International Linear Collider (ILC). This process has previously been analyzed in an EFT formalism, using LEP and LHC results, in Refs. [21,22]. In Sec. IV, we discuss the effect of the expected measurements of Higgs branching ratios to $\gamma \gamma, \gamma Z$, and $\mu^{+} \mu^{-}$at the LHC.

In Sec. V, we explain how the measurement of the cross section, angular distribution, and polarization asymmetry for $e^{+} e^{-} \rightarrow Z h$ constrain the EFT parameters. An EFT analysis of the total cross section for this process has previously been given in Ref. [5]. We will show that these measurements supply the missing pieces of information needed to constrain the full set of nine operators responsible for new physics effects in vector-boson, lepton, and Higgs couplings.

In principle, this should be enough information to extract the triple Higgs coupling parameter $c_{6}$ from the measurement of the cross section for $e^{+} e^{-} \rightarrow Z h h$. However, in practice, the constraint turns out not to be strong enough. We can find additional constraints on the EFT parameters by studying the other major single Higgs production process available at $e^{+} e^{-}$colliders: the $W$ fusion process $e^{+} e^{-} \rightarrow \nu \bar{\nu} h$. This reaction has a larger cross section than $e^{+} e^{-} \rightarrow Z h$ at $500 \mathrm{GeV}$, and it also depends strongly on the EFT parameters. However, in this case, there is no specific Higgs boson tag and so the cross section cannot be measured in a model-independent way. To make use of this process, we will need also to study the Higgs decay partial widths. These also have expansions in EFT parameters. These bring in another seven parameters beyond our original set, but in the end, all of the parameters can be strongly constrained.

Thus, in Sec. VI, we work out formulas in terms of EFT parameters for the total cross section for $e^{+} e^{-} \rightarrow \nu \bar{\nu} h$. In Sec. VII, we present the EFT formulas for the various Higgs boson partial widths. This formalism provides the basis not only to determine the shift of the triple Higgs coupling but also to develop a method for determining the full set of Higgs boson couplings in a model-independent way. The implications of this formalism for Higgs coupling determination at $e^{+} e^{-}$colliders will be presented in a companion paper [23].

Finally, in Sec. VIII, we present the dependence of the cross section for $e^{+} e^{-} \rightarrow$ Zhh in terms of our full set of 
parameters. We estimate the error on the prediction of the total cross section for this reaction due to uncertainties from all new physics effects except for the variation of the triple Higgs coupling. This estimate makes use of projections for the accuracy of high-precision measurements of single Higgs processes expected to be carried out at the ILC [24-26]. We estimate that this uncertainty in the total cross section will be $2.4 \%$, corresponding to a $5 \%$ systematic uncertainty in the determination of the triple Higgs coupling. This is attractively small and should be subdominant to expected statistical and direct experimental systematic errors.

Section IX presents our conclusions. Appendix A summarizes the formulas used in our fit. Appendix B specifies the inputs to the fit in more detail.

\section{EFFECTIVE FIELD THEORY FORMALISM}

In this paper, we represent the effects of new physics by writing an extension of the SM as an effective field theory. The SM is already the most general theory with operators of dimension four or lower, $S U(3) \times S U(2) \times U(1)$ gauge invariance, the known spectrum of quarks and leptons, and one $S U(2)$-doublet Higgs field. If new physics effects are due to new heavy particles of mass at least $M$, their effects can be represented by adding operators of dimension six. The effects of these operators are suppressed by factors $1 / M^{2}$. For $M>500 \mathrm{GeV}$, as suggested by LHC results, these factors already push the size of the most general new physics effects below the current sensitivity of LHC Higgs measurements. Effects of operators of dimension eight and higher are suppressed by additional powers of $1 / M^{2}$, and we will neglect them in this discussion.

The restriction to dimension-six operator perturbations leaves a great deal of freedom. For the SM with one fermion generation, there are a total of 84 independent dimensionsix operators that can be added to the Lagrangian. Of these, eight are baryon-number violating and, of the remainder, 59 are $C P$ conserving while 17 are $C P$ violating $[27,28]$. Fortunately, not all of these operators contribute to the processes of interest in a given study. For the goals of this paper, a subset of 17 of these operators will suffice for a general analysis. These are divided into a set of ten governing vector-boson, lepton, and Higgs boson couplings and another set of seven, which will be introduced in Sec. VII, needed for other possible Higgs decays.

\section{A. Operator basis}

One aspect of the study of the dimension-six effective operators is that there are many possible choices of basis. In this paper, we will study processes that involve only light leptons, electroweak gauge bosons, and Higgs bosons. Thus, we should choose an operator basis that is convenient for analyzing this particular system. We choose a basis that includes the minimum number of operators that include only gauge fields and Higgs fields, using the equations of motion to convert purely bosonic operators to operators that include quark and lepton fields. Some operators that involve the lepton fields must also be included in the analysis. The use of equations of motion to make these reductions and other aspects of the EFT formalism were explained in Refs. [27,29-32] and many other papers. A very convenient choice for our analysis is the "Warsaw" basis put forward in Ref. [27]. In the $C P$-conserving case, this basis contains only seven operators containing only the $W, Z$, and Higgs boson fields, and another three relevant operators containing lepton fields. We will slightly rearrange the pure Higgs operators, as is done in the "SILH" basis $[3,4]$, for convenience in the analysis. In the $C P$ violating case, another four operators need to be included.

In this section, we will present the basic formalism and notation for these operators. We generally follow the conventions of Ref. [33], which in turn are based on Refs. [3,4]. The same basis was used (with slightly different notation) in Ref. [5].

Our analysis will use ten $C P$-conserving operators from the Warsaw basis of dimension-six operators. We will denote these as

$$
\begin{aligned}
& \Delta \mathcal{L}=\frac{c_{H}}{2 v^{2}} \partial^{\mu}\left(\Phi^{\dagger} \Phi\right) \partial_{\mu}\left(\Phi^{\dagger} \Phi\right)+\frac{c_{T}}{2 v^{2}}\left(\Phi^{\dagger} \stackrel{\leftrightarrow}{D} \mu \mid\left(\Phi^{\dagger} \stackrel{\leftrightarrow}{D}_{\mu} \Phi\right)-\frac{c_{6} \lambda}{v^{2}}\left(\Phi^{\dagger} \Phi\right)^{3}+\frac{g^{2} c_{W W}}{m_{W}^{2}} \Phi^{\dagger} \Phi W_{\mu \nu}^{a} W^{a \mu \nu}+\frac{4 g g^{\prime} c_{W B}}{m_{W}^{2}} \Phi^{\dagger} t^{a} \Phi W_{\mu \nu}^{a} B^{\mu \nu}\right. \\
& +\frac{g^{\prime 2} c_{B B}}{m_{W}^{2}} \Phi^{\dagger} \Phi B_{\mu \nu} B^{\mu \nu}+\frac{g^{3} c_{3 W}}{m_{W}^{2}} \epsilon_{a b c} W_{\mu \nu}^{a} W_{\rho}^{b \nu} W^{c \rho \mu}+i \frac{c_{H L}}{v^{2}}\left(\Phi^{\dagger} \stackrel{\leftrightarrow}{D}^{\mu} \Phi\right)\left(\bar{L} \gamma_{\mu} L\right)+4 i \frac{c_{H L}^{\prime}}{v^{2}}\left(\Phi^{\dagger} t^{a} \stackrel{\leftrightarrow}{D} \mu\right)\left(\bar{L} \gamma_{\mu} t^{a} L\right) \\
& +i \frac{c_{H E}}{v^{2}}\left(\Phi^{\dagger} \stackrel{\leftrightarrow}{D}^{\mu} \Phi\right)\left(\bar{e} \gamma_{\mu} e\right)
\end{aligned}
$$

The parameter $c_{6}$ shifts the Higgs potential. The other parameters express different possible new physics effects. The operators in Eq. (1) must be defined at a specific momentum scale. We take this scale to be close to $500 \mathrm{GeV}$.

The four dimension-six $C P$-violating operators can be written as

$$
\Delta \mathcal{L}_{C P}=+\frac{g^{2} \tilde{c}_{W W}}{m_{W}^{2}} \Phi^{\dagger} \Phi W_{\mu \nu}^{a} \tilde{W}^{a \mu \nu}+\frac{4 g g^{\prime} \tilde{c}_{W B}}{m_{W}^{2}} \Phi^{\dagger} t^{a} \Phi W_{\mu \nu}^{a} \tilde{B}^{\mu \nu}+\frac{g^{2} \tilde{c}_{B B}}{m_{W}^{2}} \Phi^{\dagger} \Phi B_{\mu \nu} \tilde{B}^{\mu \nu}+\frac{g^{3} \tilde{c}_{3 W}}{m_{W}^{2}} \epsilon_{a b c} W_{\mu \nu}^{a} W^{b \nu} \tilde{W}^{c \rho \mu}
$$


Operators involving gluon fields are not needed for our analysis, and so do not appear in Eqs. (1) and (2). All of the parameters $c_{i}$ and $\tilde{c}_{i}$ are dimensionless.

Other bases for the dimension-six operators include additional bosonic operators called $\mathcal{O}_{W}$ and $\mathcal{O}_{B}$. When these operators are eliminated using the equations of motion, the operators $\mathcal{O}_{H L}, \mathcal{O}_{H L}^{\prime}$, and $\mathcal{O}_{H E}$, with a Higgs current and a lepton current, are generated. These terms containing lepton fields play an surprisingly important role in our analysis. They cannot be ignored.

The notation of these equations requires some explanation. $W_{\mu \nu}^{a}$ and $B_{\mu \nu}$ are the Yang-Mills field-strength tensors for $S U(2)$ and $U(1) . D_{\mu}$ is the gauge-covariant derivative, $t^{a}=\sigma^{a} / 2$, and

$$
\begin{aligned}
\Phi^{\dagger} \stackrel{\leftrightarrow}{D}_{\mu} \Phi & =\Phi^{\dagger} D_{\mu} \Phi-D_{\mu} \Phi^{\dagger} \Phi, \\
\Phi^{\dagger} t^{a} \stackrel{\leftrightarrow}{D}_{\mu} \Phi & =\Phi^{\dagger} t^{a} D_{\mu} \Phi-D_{\mu} \Phi^{\dagger} t^{a} \Phi .
\end{aligned}
$$

The tilded field strengths in Eq. (2) are

$$
\tilde{W}_{\mu \nu}^{a}=\frac{1}{2} \epsilon_{\mu \nu \lambda \sigma} W^{a \lambda \sigma}, \quad \tilde{B}_{\mu \nu}=\frac{1}{2} \epsilon_{\mu \nu \lambda \sigma} B^{\lambda \sigma} .
$$

Finally, we will write

$$
s_{w}^{2}=\sin ^{2} \theta_{w}=\frac{g^{\prime 2}}{g^{2}+g^{\prime 2}}, \quad c_{w}^{2}=\cos ^{2} \theta_{w}=\frac{g^{2}}{g^{2}+g^{\prime 2}},
$$

in terms of the $S U(2) \times U(1)$ couplings in the Lagrangian. The renormalization prescription that we will use for the couplings and the weak mixing angle will be given in Sec. II C below.

\section{B. Simplifications}

Our analysis will include a number of simplifications that we will now enumerate. None of these simplifications has a significant effect on our final answers. We will explain how the analysis given here can be systematically improved to relax some of these simplifying assumptions.

First, we will work at the tree level and strictly to linear order in the dimension-six operator coefficients. For the central values in the fit, we will assume that the SM is precisely valid and the EFT coefficients in Eq. (1) are zero.

We will primarily be concerned with the sensitivity of $e^{+} e^{-}$experiments to values of the $c_{i}$ of $1 \%$ and below. It is possible that some of the $c_{i}$ could be larger, even of order 1 , consistent with current data. That would be a wonderful situation. But in this paper we are trying to probe the limits of sensitivity of future experiments. We estimate corrections to the linear approximation in the $c_{i}$ in a manner consistent with this viewpoint. That is, effects quadratic in the $c_{i}$ should be of order $10^{-4}$, effects due to operators of dimension eight should be of order $10^{-4}$, and effects of electroweak radiative corrections to the terms linear in the $c_{i}$, including operator mixing of dimension-six operators, should be of order $\alpha_{w} / \pi \cdot 1 \%$. Then we may neglect effects of all three of these types in the analysis presented here.

It has been pointed out in Ref. [20] that some terms of order $c_{I}$ are enhanced by factors proportional to $s / m_{Z}^{2}$. We will see such enhancements appearing in our analysis. However, we will see that the uncertainties on the corresponding EFT coefficients are extremely small, such that the corrections that include these enhancement factors are still restricted to be of order 1\%. Explicit examples will be discussed in Secs. V and VIII.

Since we are concerned with such small corrections to the Standard Model predictions for Higgs cross sections and decay rates, comparisons to data should use highprecision Standard Model calculations of the cross sections and rates. In this paper, we will compute Standard Model rates only to the tree level. This will suffice for estimating the sensitivity to the new physics corrections that we consider in this paper. An actual experimental analysis will need to combine our formulas with Standard Model predictions computed at least to one-loop order, and, in most cases, to two-loop order, in electroweak corrections.

Our analysis will involve all coefficients in Eq. (1) other than the coefficient $c_{6}$ that shifts the triple Higgs coupling. In this analysis, we will not need to assume that $c_{6}$ is small, though we will ignore effects of $c_{6}$ in loop diagrams [proportional to $\lambda^{2} c_{6} /(4 \pi)^{2}$ ]. This is important to note, because models of electroweak baryogenesis expect values of $c_{6}$ of order 1 [8], and the expected error on $c_{6}$ from the ILC is $27 \%$ [12,34]. If $c_{6}$ were indeed of order $1 \%$, along with the other $c_{i}$ coefficients, its effect would not be measurable at the ILC or at any other proposed collider. One might ask if it is consistent to have $c_{6}$ of order 1 while the other EFT coefficients are extremely small. Some examples of models with this property were given in Refs. [17,35,36], and in Sec. 2.3 of Ref. [19]. More likely, corrections from the new physics that modifies $c_{6}$ will also shift the parameters such as $c_{H}$ and $c_{W W}$ in Eq. (1) to nonzero values of order a few percent. These shifts might be the first indication of a correction to the Higgs sector Lagrangian. The shifts will not affect our error estimate for $c_{6}$, though they will of course alter the value of $c_{6}$ that is extracted from the cross section for double Higgs production.

Second, we will ignore some possible dimension-six operator corrections involving the light leptons. We will consider the three coefficients $c_{H L}, c_{H L}^{\prime}, c_{H E}$ as independent free parameters. Taking this prescription, we are explicitly not assuming that the dimension-six corrections are "oblique" (in the language of Ref. [37]) or "universal" (in the language of Ref. [29]). However, we will assume electron-muon-tau universality. We will need, first, the constraint $c_{H L}^{\prime}(\mu)=c_{H L}^{\prime}(e)$, to use $G_{F}$ together with 
constraints from precision electroweak measurements involving electrons only. This assumption can be tested by measuring the equality of the $W$ boson branching ratios to $\mu$ and $e$ using the sample of almost $3 \times 10^{7} \mathrm{~W}$ pairs available at the $500 \mathrm{GeV}$ ILC. We will also use the equality of the $Z$ left-right asymmetry $A_{\ell}$ for $e$ and $\tau$, since we will use a value of $A_{\ell}$ with contributions from $A_{e}$ and $A_{\tau}$. (Dropping this equality has only a minor effect on our results.) This assumption can be tested through measurements of $e^{+} e^{-} \rightarrow \ell^{+} \ell^{-}$at $500 \mathrm{GeV}$. A more complete Lagrangian would also include a four-fermion operator due to new physics that contributes directly to $G_{F}$. However, this operator is already constrained to have a $\Lambda$ scale above 8.5 TeV by LEP 2 data [38], and this constraint will become much stronger when data on $e^{+} e^{-} \rightarrow \mu^{+} \mu^{-}$at $500 \mathrm{GeV}$ becomes available. In our discussion of the vector-boson, lepton, and Higgs interactions, we will also ignore leptonic terms that are mass suppressed, including the dimensionsix operators that correct the lepton-Higgs couplings and lepton-gauge boson magnetic moment couplings. The lepton terms that correct the Higgs couplings will appear in Sec. VII. A more general analysis could incorporate more of these additional parameters and the reactions that constrain them.

In this paper, we will avoid observables that involve the operators similar to the last two lines of Eq. (1) that include quark currents. There is a very large number of these operators, two for each quark flavor. Eventually, in Sec. VII, we will need to consider these operators, but only in two specific linear combinations. When we refer to these operators later in the paper, we will call the corresponding coefficients $c_{X}, c_{X}^{\prime}$.

Third, we will ignore the effects of the $C P$-violating operators in Eq. (2). We will consider only $C P$-invariant observables, and so the effects of these operators on our observables will be of order $c_{I}^{2}$. Actually, it is possible to constrain the coefficients $\tilde{c}_{W B}$ and $\tilde{c}_{3 W}$ below the percent level through the study of $e^{+} e^{-} \rightarrow W^{+} W^{-}$[39] and to constrain $\tilde{c}_{W W}$ and $\tilde{c}_{B B}$ to the few-percent level through constraints from $h \rightarrow \gamma \gamma$ and $e^{+} e^{-} \rightarrow Z h$. We will present these latter constraints in Secs. IV and V. At this level, these coefficients would give negligible contributions to our analysis.

\section{On-shell renormalization}

We then restrict ourselves to the SM Lagrangian plus the perturbation (1), considered in linear order. Our analysis of vector-boson, lepton, and Higgs couplings then contains 14 parameters-the four SM parameters, which we will take to be $g, g^{\prime}, v$, and a Higgs coupling $\bar{\lambda}$, and the ten parameters in Eq. (1) (including $c_{6}$ ). The dimension-six operator coefficients alter the SM expressions for precision electroweak observables and thus shift the appropriate values for the Standard Model couplings. In our analysis, we will deal with this by allowing the shifts of $g, g^{\prime}, v$ and $\bar{\lambda}$ from their
SM values to be free parameters in our fit. In this tree-level analysis, it is useful to think of $g$ and the other couplingsand the parameters $s_{w}$ and $c_{w}$-as bare values set by fitting an expression that includes the SM expectations and corrections perturbative in the $c_{i}$ to a set of measurements. This defines an on-shell renormalization procedure.

Using the notation

$$
\delta A=\frac{\Delta A}{A},
$$

we will write expressions for the deviation of observables from their SM predictions as linear combinations of the coefficients $c_{I}$ and the deviations (6) of the SM parameters. A list of all of the expressions of this type entering our fit is given in Appendix A.

Another approach to on-shell renormalization is given by the $S, T$ formalism [37]. We will sketch the formulas for $S, T$ renormalization with EFT parameters in Appendix C.

The operators (1) also renormalize the kinetic terms of the SM fields. The contributions in Eq. (1) give shifts of the SM kinetic terms

$$
\begin{aligned}
\mathcal{L}= & -\frac{1}{2} W_{\mu \nu}^{+} W^{-\mu \nu} \cdot\left(1-\delta Z_{W}\right)-\frac{1}{4} Z_{\mu \nu} Z^{\mu \nu} \cdot\left(1-\delta Z_{Z}\right) \\
& -\frac{1}{4} A_{\mu \nu} A^{\mu \nu} \cdot\left(1-\delta Z_{A}\right)+\frac{1}{2}\left(\partial_{\mu} h\right)\left(\partial^{\mu} h\right) \cdot\left(1-\delta Z_{h}\right),
\end{aligned}
$$

with

$$
\begin{aligned}
\delta Z_{W} & =\left(8 c_{W W}\right), \\
\delta Z_{Z} & =c_{w}^{2}\left(8 c_{W W}\right)+2 s_{w}^{2}\left(8 c_{W B}\right)+s_{w}^{4} / c_{w}^{2}\left(8 c_{B B}\right), \\
\delta Z_{A} & =s_{w}^{2}\left(\left(8 c_{W W}\right)-2\left(8 c_{W B}\right)+\left(8 c_{B B}\right)\right), \\
\delta Z_{h} & =-c_{H} .
\end{aligned}
$$

We will rescale the boson fields to remove these factors from the kinetic terms. Then the $\delta Z$ factors will appear in the vertices that we write below. The field-strength renormalization for the Higgs field, proportional to $c_{H}$, plays a key role in our analysis and in the general theory of Higgs couplings [40,41]. It is important to note that the mass eigenstates $Z$ and $A$ are not altered by the addition of Eq. (1). The $c_{T}$ term shifts the mass of the $Z$ eigenstate without mixing it with the $A$. However, Eq. (1) does induce a kinetic mixing between $Z$ and $A$,

$$
\Delta \mathcal{L}=\frac{1}{2} \delta Z_{A Z} A_{\mu \nu} Z^{\mu \nu},
$$

with 
$\delta Z_{A Z}=s_{w} c_{w}\left(\left(8 c_{W W}\right)-\left(1-\frac{s_{w}^{2}}{c_{w}^{2}}\right)\left(8 c_{W B}\right)-\frac{s_{w}^{2}}{c_{w}^{2}}\left(8 c_{B B}\right)\right)$.

We will treat this effect in perturbation theory.

The masses of the bosons are then given by

$$
\begin{aligned}
m_{W}^{2} & =\frac{g^{2} v^{2}}{4}\left(1+\delta Z_{W}\right), \\
m_{Z}^{2} & =\frac{\left(g^{2}+g^{2}\right) v^{2}}{4}\left(1-c_{T}+\delta Z_{Z}\right), \\
m_{h}^{2} & =2 \bar{\lambda} v^{2}\left(1+\delta Z_{h}\right)
\end{aligned}
$$

where

$$
\bar{\lambda}=\lambda\left(1+\frac{3}{2} c_{6}\right)
$$

It is useful to take $\bar{\lambda}$ as a basic coupling, since the Higgs quartic coupling $\lambda$ and the dimension-six coefficient $c_{6}$ appear only in this combination until we actually encounter the triple Higgs coupling in our analysis. The formulas (11) are not precise for the absolute values of the masses without the inclusion of loop corrections. However, the differential relations

$$
\begin{aligned}
\delta m_{W} & =\delta g+\delta v+\frac{1}{2} \delta Z_{W}, \\
\delta m_{Z} & =c_{w}^{2} \delta g+s_{w}^{2} \delta g^{\prime}+\delta v-\frac{1}{2} c_{T}+\frac{1}{2} \delta Z_{Z}, \\
\delta m_{h} & =\frac{1}{2} \delta \bar{\lambda}+\delta v+\frac{1}{2} \delta Z_{h}
\end{aligned}
$$

are accurate for small deviations.

To expand other precision electroweak observables, it is useful to expand expressions built from the bare couplings

$$
\begin{aligned}
& \delta s_{w}=-c_{w}^{2}\left(\delta g-\delta g^{\prime}\right), \\
& \delta c_{w}=s_{w}^{2}\left(\delta g-\delta g^{\prime}\right) .
\end{aligned}
$$

The physical electric charge is expanded as

$$
\delta e=\delta\left(4 \pi \alpha\left(m_{Z}^{2}\right)\right)^{1 / 2}=s_{w}^{2} \delta g+c_{w}^{2} \delta g^{\prime}+\frac{1}{2} \delta Z_{A} .
$$

The Fermi constant obtains a contribution from one of the Higgs-lepton current-current operators. It also receives contributions $\left(1+\delta Z_{W}\right)$ from the $W$ mass and coupling that cancel between the numerator and denominator. Then

$$
\delta G_{F}=1-2 \delta v+2 c_{H L}^{\prime} .
$$

In writing the $Z$ boson couplings of the light leptons, it is convenient to include the contribution due to the $A Z$ kinetic

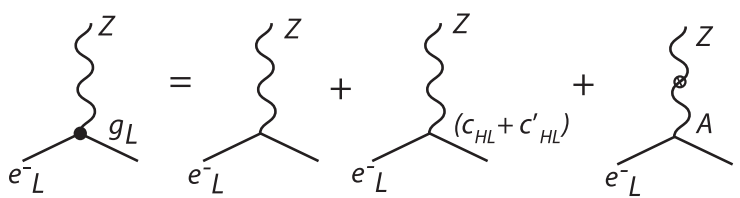

FIG. 1. Contributions to $g_{L}$, the left-handed electron coupling to the $Z$, including the effects of contact interactions and $A Z$ kinetic mixing. The contributions to $g_{R}$ have a similar structure.

mixing in Eq. (9), as shown in Fig. 1. Then the left- and right-handed charged lepton couplings are

$$
\begin{aligned}
g_{L}= & \frac{g}{c_{w}}\left[\left(-\frac{1}{2}+s_{w}^{2}\right)\left(1+\frac{1}{2} \delta Z_{Z}\right)-\frac{1}{2}\left(c_{H L}+c_{H L}^{\prime}\right)\right. \\
& \left.-s_{w} c_{w} \delta Z_{A Z}\right], \\
g_{R}= & \frac{g}{c_{w}}\left[\left(+s_{w}^{2}\right)\left(1+\frac{1}{2} \delta Z_{Z}\right)-\frac{1}{2} c_{H E}-s_{w} c_{w} \delta Z_{A Z}\right] .
\end{aligned}
$$

The $W$ coupling to leptons is given by

$$
g_{W}=g\left(1+c_{H L}^{\prime}+\frac{1}{2} \delta Z_{W}\right)
$$

In Sec. II E, we will introduce $Z$ coupling to $W^{+} W^{-}$. Its value is

$$
g_{Z}=g c_{w}\left(1+\frac{1}{2} \delta Z_{Z}+\frac{s_{w}}{c_{w}} \delta Z_{A Z}\right)
$$

The differentials of these expressions are written in Appendix A.

\section{Precision electroweak constraints}

The five parameters $m_{W}, m_{Z}, m_{h}, \alpha\left(m_{Z}\right), G_{F}$ constrain independent combinations of the four Standard Model couplings and the dimension-six coefficients. Most of the power of the precision electroweak constraints on our parameter set is given by adding two further, very precise, measurements from $Z$ physics. We choose these to be $\Gamma_{\ell}$, the partial width of the $Z$ to a lepton, and $A_{\ell}$, the left-right asymmetry of the $Z$ coupling to leptons. All dimension-six corrections to these coefficients are already incorporated into $g_{L}$ and $g_{R}$, so the differentials of these parameters are given in terms of Eq. (17) by

$$
\begin{aligned}
\delta \Gamma_{\ell} & =\delta m_{Z}+2 \frac{g_{L}^{2} \delta g_{L}+g_{R}^{2} \delta g_{R}}{g_{L}^{2}+g_{R}^{2}}, \\
\delta A_{\ell} & =\frac{4 g_{L}^{2} g_{R}^{2}\left(\delta g_{L}-\delta g_{R}\right)}{g_{L}^{4}-g_{R}^{4}} .
\end{aligned}
$$


TABLE I. Values and uncertainties for precision electroweak observables used in this paper. The values are taken from Ref. [42], except for the averaged value of $A_{\ell}$, which corresponds to the averaged value of $\sin ^{2} \theta_{\text {eff }}$ in Ref. [43]. The best -fit values are those of the fit in Ref. [42]. For the purpose of fitting Higgs boson couplings as described in Sec. VII, we use improvements in some of the errors expected from the LHC [44] and ILC [45]. The improved estimate of the $W$ width is obtained from $\Gamma_{W}=\Gamma(W \rightarrow \ell \nu) / \mathrm{BR}(W \rightarrow \ell \nu)$.

\begin{tabular}{lcccc}
\hline \hline Observable & Current value & Current $\sigma$ & Future $\sigma$ & SM best-fit value \\
\hline$\alpha^{-1}\left(m_{Z}^{2}\right)$ & 128.9220 & 0.0178 & & (same) \\
$G_{F}\left(10^{-10} \mathrm{GeV}^{-2}\right)$ & 1166378.7 & 0.6 & & $($ same $)$ \\
$m_{W}(\mathrm{MeV})$ & 80385 & 15 & 5 & 80361 \\
$m_{Z}(\mathrm{MeV})$ & 91187.6 & 2.1 & & 91188.0 \\
$m_{h}(\mathrm{MeV})$ & 125090 & 240 & 15 & 125110 \\
$A_{\ell}$ & 0.14696 & 0.0013 & & 0.147937 \\
$\Gamma_{\ell}(\mathrm{MeV})$ & 83.984 & 0.086 & & 83.995 \\
$\Gamma_{Z}(\mathrm{MeV})$ & 2495.2 & 2.3 & 2 & 2494.3 \\
$\Gamma_{W}(\mathrm{MeV})$ & 2085 & 42 & 2 & 2088.8 \\
\hline \hline
\end{tabular}

Note that no dimension-six operators involving quarks enter an analysis based on these observables.

The values that we will use here for the precision electroweak observables, and their errors, are the current values, from Ref. [42]. For the $A_{\ell}$, we take the value corresponding to the average of $\sin ^{2} \theta_{\text {eff }}^{\text {lept }}$ presented in Sec. 7.3.4 of Ref. [43]. These values are shown in Table I.

For the analysis in Sec. VII, we will need to make use of the measurements of the total width of the $Z$ and $W$. So we include those current values also in Table I.

Our analysis will benefit from improvements in some of the precision electroweak parameters that we expect to see in the era of $e^{+} e^{-}$experiments. The uncertainties on $m_{W}$ measurements are expected to be improved to $5 \mathrm{MeV}$ already at the LHC [44]. The ILC is expected to improve the error on the Higgs boson mass to $15 \mathrm{MeV}$ by recoil mass fitting of $e^{+} e^{-} \rightarrow Z h$ events in which the $Z$ decays to leptons [45]. It is not so easy to obtain a very precise direct measurement of the $W$ total width. Today, this width is known only to $2 \%$ accuracy. However, with the use of constraints from other precision electroweak observables and measurements of $e^{+} e^{-} \rightarrow W^{+} W^{-}$, our EFT formalism predicts the partial width $\Gamma(W \rightarrow \ell \nu)$ to an accuracy of $0.06 \%$. Using the large statistics available at the ILC $-3 \times 10^{7}$ pairs - it will be possible to apply a tagand-probe method to make a very precise measurement of the branching ratio $\mathrm{BR}(W \rightarrow \ell \nu)$. We then expect that the total width $\Gamma_{W}$ can be known to better than $0.1 \%$. Running an $e^{+} e^{-}$collider at the $Z$ resonance to create at least $10^{9} Z$ bosons would be expected to improve the errors on $A_{\ell}$ and $\Gamma_{\ell}$ by an order of magnitude [46]. However, we will not make use of that possibility in the estimates given in this paper.

\section{E. $W, Z$, and Higgs boson vertices}

Starting from the Lagrangian (1) and using the prescriptions in Sec. II C, we can work out expressions for the various coupling constants that appear in the $W$ and Higgs interactions.

The three-boson vertices involving the $W$ boson are canonically written [47]

$$
\begin{aligned}
\Delta \mathcal{L}_{T G C}= & i g_{V}\left\{V^{\mu}\left(\hat{W}_{\mu \nu}^{-} W^{+\nu}-\hat{W}_{\mu \nu}^{+} W^{-\nu}\right)+\kappa_{V} W_{\mu}^{+} W_{\nu}^{-} \hat{V}^{\mu \nu}\right. \\
& \left.+\frac{\lambda_{V}}{m_{W}^{2}} \hat{W}_{\mu}^{-\rho} \hat{W}_{\rho \nu}^{+} \hat{V}^{\mu \nu}\right\}
\end{aligned}
$$

where $V=A$ or $Z$ and

$$
\hat{V}_{\mu \nu}=\partial_{\mu} V_{\nu}-\partial_{\nu} V_{\mu}
$$

is the linear part (only) of the field-strength tensor. Note that we have absorbed the constant in front of the first term in Eq. (21) into the overall coupling $g_{A}$ or $g_{Z}$. Then this formula has six parameters. Of these $g_{A}$ must be equal to the physical electron charge $e$ in Eq. (15), since this is also the charge of the $W$. It is a simple exercise to verify this explicitly. We define the charge $g_{Z}$ to include the effect of $A Z$ kinetic mixing, as shown in Fig. 2. Then the charge $g_{Z}$ is given by

$$
g_{Z}=g c_{w}\left(1+\frac{1}{2} \delta Z_{Z}+\frac{s_{w}}{c_{w}} \delta Z_{A Z}\right) .
$$

The remaining parameters are given by

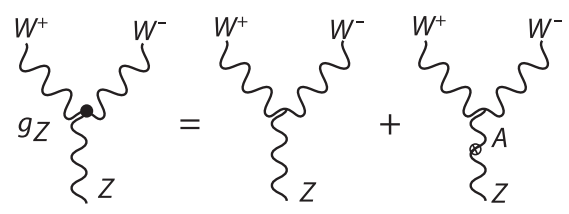

FIG. 2. Contributions to $g_{Z}$, the coupling of the $Z$ boson to $W^{+} W^{-}$, including in particular the effect of $A Z$ kinetic mixing. 


$$
\begin{aligned}
& \kappa_{A}=1+\left(8 c_{W B}\right), \\
& \kappa_{Z}=1-\frac{s_{w}^{2}}{c_{w}^{2}}\left(8 c_{W B}\right), \\
& \lambda_{A}=\lambda_{Z}=-6 g^{2} c_{3 W} .
\end{aligned}
$$

Because of the relations between these expressions, the measurement of the $W W A$ and $W W Z$ vertices contribute three (not five) additional constraints on our 14 variables. We will work out the form of these constraints in Sec. III.

In a similar way, we will write the Lagrangian for the Higgs boson and its coupling to vector bosons in a canonical form as

$$
\begin{aligned}
\Delta \mathcal{L}_{h}= & \frac{1}{2} \partial_{\mu} h \partial^{\mu} h-\frac{1}{2} m_{h}^{2} h^{2}-\left(1+\eta_{h}\right) \bar{\lambda} v h^{3}+\frac{\theta_{h}}{v} h \partial_{\mu} h \partial^{\mu} h \\
& +\left(1+\eta_{W}\right) \frac{2 m_{W}^{2}}{v} W_{\mu}^{+} W^{-\mu} h+\left(1+\eta_{W W}\right) \frac{m_{W}^{2}}{v^{2}} W_{\mu}^{+} W^{-\mu} h^{2} \\
& +\left(1+\eta_{Z}\right) \frac{m_{Z}^{2}}{v} Z_{\mu} Z^{\mu} h+\frac{1}{2}\left(1+\eta_{Z Z}\right) \frac{m_{Z}^{2}}{v^{2}} Z_{\mu} Z^{\mu} h^{2} \\
& +\zeta_{W} \hat{W}_{\mu \nu}^{+} \hat{W}^{-\mu \nu}\left(\frac{h}{v}+\frac{1}{2} \frac{h^{2}}{v^{2}}\right)+\frac{1}{2} \zeta_{Z} \hat{Z}_{\mu \nu} \hat{Z}^{\mu \nu}\left(\frac{h}{v}+\frac{1}{2} \frac{h^{2}}{v^{2}}\right) \\
& +\frac{1}{2} \zeta_{A} \hat{A}_{\mu \nu} \hat{A}^{\mu \nu}\left(\frac{h}{v}+\frac{1}{2} \frac{h^{2}}{v^{2}}\right)+\zeta_{A Z} \hat{A}_{\mu \nu} \hat{Z}^{\mu \nu}\left(\frac{h}{v}+\frac{1}{2} \frac{h^{2}}{v^{2}}\right) .
\end{aligned}
$$

The six parameters in the first two lines of this equation are given to first order in the EFT coefficients by

$$
\begin{aligned}
\eta_{h} & =\delta \bar{\lambda}+\delta v-\frac{3}{2} c_{H}+c_{6}, \\
\theta_{h} & =c_{H}, \\
\eta_{W} & =2 \delta m_{W}-\delta v-\frac{1}{2} c_{H}, \\
\eta_{W W} & =2 \delta m_{W}-2 \delta v-c_{H}, \\
\eta_{Z} & =2 \delta m_{Z}-\delta v-\frac{1}{2} c_{H}-c_{T}, \\
\eta_{Z Z} & =2 \delta m_{Z}-2 \delta v-c_{H}-5 c_{T} .
\end{aligned}
$$

The four parameters in the last two lines are given by

$$
\begin{aligned}
\zeta_{W}= & \delta Z_{W}=\left(8 c_{W W}\right), \\
\zeta_{Z}= & \delta Z_{Z}=c_{w}^{2}\left(8 c_{W W}\right)+2 s_{w}^{2}\left(8 c_{W B}\right)+\frac{s_{w}^{4}}{c_{w}^{2}}\left(8 c_{B B}\right), \\
\zeta_{A}= & \delta Z_{A}=8 s_{w}^{2}\left(\left(8 c_{W W}\right)-2\left(8 c_{W B}\right)+\left(8 c_{B B}\right)\right), \\
\zeta_{A Z}= & \delta Z_{A Z}=s_{w} c_{w}\left(\left(8 c_{W W}\right)-\left(1-\frac{s_{w}^{2}}{c_{w}^{2}}\right)\left(8 c_{W B}\right)\right. \\
& \left.-\frac{s_{w}^{2}}{c_{w}^{2}}\left(8 c_{B B}\right)\right) .
\end{aligned}
$$

It is important to note that Eq. (25) contains a second structure for the triple Higgs vertex, with coefficient $\theta_{h}$. In double Higgs production, this term cannot be separated from the Standard Model triple Higgs coupling except by high statistics measurements of the $m(h h)$ distribution. In our analysis, this contribution will be fixed by the determination of $c_{H}$ through precision measurements of single Higgs production.

The Lagrangian (1) also contains contact interactions between the $Z$, Higgs, and lepton fields,

$$
\begin{aligned}
\Delta \mathcal{L}_{e e h Z} \\
=-\frac{g}{2 c_{w}}\left(c_{H L}-c_{H L}^{\prime}\right)\left(\bar{\nu}_{L} \gamma_{\mu} \nu_{L}\right) Z^{\mu}\left(1+2 \frac{h}{v}+\frac{h^{2}}{v^{2}}\right) \\
-\frac{g}{2 c_{w}}\left(c_{H L}+c_{H L}^{\prime}\right)\left(\bar{e}_{L} \gamma_{\mu} e_{L}\right) Z^{\mu}\left(1+2 \frac{h}{v}+\frac{h^{2}}{v^{2}}\right) \\
-\frac{g}{2 c_{w}}\left(c_{H E}\right)\left(\bar{e}_{R} \gamma_{\mu} e_{R}\right) Z^{\mu}\left(1+2 \frac{h}{v}+\frac{h^{2}}{v^{2}}\right) \\
+\frac{g}{\sqrt{2}}\left(c_{H L}^{\prime}\right)\left(\bar{e}_{L} \gamma_{\mu} \nu_{L} W^{-\mu}+\bar{\nu}_{L} \gamma_{\mu} e_{L} W^{+\mu}\right)\left(1+2 \frac{h}{v}+\frac{h^{2}}{v^{2}}\right) .
\end{aligned}
$$

We will discuss the use of Eqs. (25) and (28) to construct expressions for the cross sections for $e^{+} e^{-} \rightarrow Z h$ and $e^{+} e^{-} \rightarrow Z h h$ in Secs. V and VI. The effects of the contact interactions in Eq. (28) on these reactions have previously been studied in Ref. [20], where it was pointed out that the coefficients typically appear in the form $c_{i} \cdot s / m_{Z}^{2}$.

\section{CONSTRAINTS FROM $e^{+} e^{-} \rightarrow W^{+} W^{-}$}

We now discuss the constraints on the dimension-six coefficients coming from measurements of $e^{+} e^{-} \rightarrow W^{+} W^{-}$. The constraints coming from the LEP and LHC experiments have been discussed already in Refs. [21,22]. However, future $e^{+} e^{-}$experiments will have additional advantages. Since $e^{+} e^{-} \rightarrow W^{+} W^{-}$is the process with the largest cross section in high-energy $e^{+} e^{-}$annihilation, very high statistics will be available. By making use especially of the mode in which one $W$ decays hadronically and one decays leptonically, the full kinematics of the $W^{+} W^{-}$production and decay can be reconstructed for each event. Changing the beam polarization from $e_{L}^{-} e_{R}^{+}$to $e_{R}^{-} e_{L}^{+}$is an order-1 effect. Using all of these handles, it is possible to probe very accurately for the effects of modifications of the Standard Model.

At the tree level, the amplitudes for $e^{+} e^{-} \rightarrow W^{+} W^{-}$are derived from the diagrams shown in Fig. 3. Typically, the predictions of these diagrams (with higher-order electroweak corrections) are compared to data by assuming that the vertices between leptons and gauge bosons have exactly the SM form while the $W W A$ and $W W Z$ vertices can contain additional terms induced by new physics. In the EFT, there are relations within the full set of 


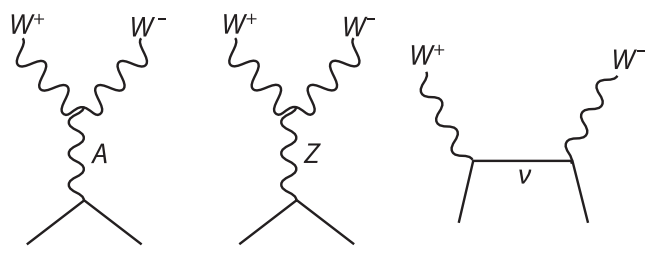

FIG. 3. Feynman diagrams contributing to the amplitudes for $e^{+} e^{-} \rightarrow W^{+} W^{-}$.

phenomenological parameters present in Eq. (21). These relations, which follow from the $S U(2) \times U(1)$ gauge invariance of the full theory [48], are written in our notation as $g_{A}=e$ and

$$
\left(\kappa_{Z}-1\right)=-\frac{s_{w}^{2}}{c_{w}^{2}}\left(\kappa_{A}-1\right), \quad \lambda_{Z}=\lambda_{A}
$$

Then the three parameters $g_{Z}, \kappa_{A}, \lambda_{A}$ are extracted from the data. The most incisive projections of the capabilities of future $e^{+} e^{-}$colliders to extract these parameters were done using this method by Marchesini [49] and Rosca [50]. These studies used full simulation with the ILD detector model at the ILC. The precise uncertainties expected at $500 \mathrm{GeV}$ with the expected $4 \mathrm{ab}^{-1}$ data sample, including their correlations, are given in Appendix B.

The assumption that the SM lepton-gauge boson vertices are unmodified is justified to a certain extent by the strength of the precision electroweak constraints on those vertices, but it is not completely consistent as an expansion in the dimension-six EFT coefficients. This point was made explicitly by Falkowski and Riva in Ref. [21], although they neglected this effect in their analysis for the practical reason that it is unimportant in fitting LEP and LHC data. For higher-accuracy measurements, one should in principle fully refit the experimental data with a formula that includes all of the terms linear in dimension-six coefficients. Here we propose a simplified treatment of this issue. It is well appreciated that the process $e^{+} e^{-} \rightarrow W^{+} W^{-}$is especially sensitive to new physics corrections because the helicity amplitudes for this process contain terms proportional to the $c_{I}$ coefficients enhanced by $s / m_{W}^{2}$. So, we will propose definitions of effective values of $g_{Z}, \kappa_{Z}$, and $\lambda_{Z}$ that agree with the standard definitions when the precision electroweak constraints are exact and otherwise include the deviations from SM precision electroweak predictions proportional to $c_{I} s / m_{W}^{2}$.

To do this, we compute the high-energy limit of the helicity amplitudes for $e^{+} e^{-} \rightarrow W^{+} W^{-}$for the case in which both $W$ bosons have longitudinal polarization. For both possible beam polarization states, the results have the form

$$
i \mathcal{M} \rightarrow-i \sin \theta \frac{s}{2 m_{W}^{2}} \mathcal{A}_{L, R}
$$

For $e_{R}^{-} e_{L}^{+}$, the neutrino diagram does not contribute and we find

$$
\mathcal{A}_{R}=e^{2} \kappa_{A}+g_{R} g_{Z} \kappa_{Z}
$$

For $e_{L}^{-} e_{R}^{+}$, all three diagrams contribute and we find

$$
\mathcal{A}_{L}=e^{2} \kappa_{A}+g_{L} g_{Z} \kappa_{Z}-\frac{g_{W}^{2}}{2} .
$$

It is easy to check that both quantities (31) and (32) vanish when the coupling constants take their SM values (including $\kappa_{A}=\kappa_{Z}=1$ ). Note that both amplitudes are independent of $\lambda_{A}$ and $\lambda_{Z}$. The $\lambda$ parameters multiply a different $s / m_{W}^{2}$ term that appears in the helicity amplitudes for the production of two transversely polarized $W$ bosons.

We propose, then, to use the following quantities to express the constraints on the $c_{I}$ from $e^{+} e^{-} \rightarrow W^{+} W^{-}$:

$$
\begin{aligned}
\left(g_{Z, \mathrm{eff}}-1\right) & =\frac{1}{g^{2} c_{w}^{2}}\left(2 \Delta \mathcal{A}_{L}-\Delta \mathcal{A}_{R}\right), \\
\left(\kappa_{A, \mathrm{eff}}-1\right) & =\frac{1}{g^{2}}\left(2 \Delta \mathcal{A}_{L}+\frac{c_{w}^{2}-s_{w}^{2}}{s_{w}^{2}} \Delta \mathcal{A}_{R}\right), \\
\lambda_{A, \mathrm{eff}} & =\lambda_{A} .
\end{aligned}
$$

The right-hand sides of Eq. (33) can be expanded in terms of the variations of SM parameters and the $c_{I}$. The expansions are written out in Appendix A. These formulas can be considered to be the quantities constrained by the analyses of Refs. [49,50]. The measurements of $W$ vertices at the LHC should be compared to similar formulas that involve the $c_{H X}$ parameters for the various quark species that participate in the observed processes.

\section{CONSTRAINTS FROM $h \rightarrow \gamma \gamma$ AND $h \rightarrow \gamma Z$}

We have now explained how to constrain ten combinations of the 14 parameters in our analysis. Before we come to precision Higgs physics in $e^{+} e^{-}$collisions, there is one more important constraint that we can apply.

The Higgs boson decays $h \rightarrow \gamma \gamma$ and $h \rightarrow Z \gamma$ receive their first SM contributions at the one-loop level. In both cases, these contributions are very small. However, both decays receive tree-level contributions from the dimensionsix Lagrangian, proportional to the coefficients $\zeta_{A}$ and $\zeta_{A Z}$ in Eq. (25). If these decays are observed to have rates close to their SM values, the parameters $\zeta_{A}$ and $\zeta_{A Z}$ are constrained to have values that are small fractions of the already suppressed SM decay amplitudes [51]. Already, the constraints from the LHC on $h \rightarrow \gamma \gamma$ are quite strong. Eventually, the measurement of these modes will provide an extremely strong constraint on the parameter $c_{B B}$ and a significant constraint on the parameter $c_{W B}$.

We now analyze this point in more detail. The $h \rightarrow \gamma \gamma$ decay amplitude has the form 


$$
i \mathcal{M}=i \mathcal{A}\left(q_{1}^{\mu} \cdot q_{2}^{\nu}-q_{1}^{\nu} q_{2}^{\mu}\right) \epsilon_{1 \mu}^{*} \epsilon_{2 \nu}^{*} .
$$

The $\zeta_{A}$ term contributes an extra factor

$$
\Delta \mathcal{A}=\frac{2 \zeta_{A}}{v} .
$$

Then

$$
\begin{aligned}
\delta \Gamma(h \rightarrow \gamma \gamma) & =4 \frac{\zeta_{A}}{v}\left[\frac{m_{h}^{3}}{\left.64 \pi \Gamma(h \rightarrow \gamma \gamma)\right|_{\mathrm{SM}}}\right]^{1 / 2} \\
& =526 \zeta_{A} .
\end{aligned}
$$

In a similar way, we find

$$
\begin{aligned}
\delta \Gamma(h \rightarrow Z \gamma) & =4 \frac{\zeta_{A Z}}{v}\left[\frac{m_{h}^{3}\left(1-m_{Z}^{2} / m_{h}^{2}\right)^{3}}{\left.32 \pi \Gamma(h \rightarrow Z \gamma)\right|_{\mathrm{SM}}}\right]^{1 / 2} \\
& =290 \zeta_{A Z} .
\end{aligned}
$$

We must add to these expressions the variation of the SM predictions for the decay widths with respect to the SM parameters. The complete expressions are given in Appendix A. We omit loop-suppressed corrections from the $c_{I}$ coefficients. In fact, Eqs. (36) and (37) are by far the dominant effects.

It is not possible to measure absolute Higgs decay widths at the LHC, because there is no strategy to obtain the total Higgs width to high accuracy. But, it is possible to measure ratios of branching ratios from which the total Higgs width cancels out. Since the measurement of each Higgs boson final state at the LHC requires its own strategy, measurements of ratios of branching ratios are typically limited by the separate systematic errors from production and detection of the two processes that are compared. The only exceptions of which we are aware are the ratios

$$
\begin{array}{ll}
\frac{\mathrm{BR}\left(h \rightarrow Z Z^{*} \rightarrow 4 \ell\right)}{\mathrm{BR}(h \rightarrow \gamma \gamma)}, & \frac{\mathrm{BR}(h \rightarrow Z \gamma)}{\mathrm{BR}(h \rightarrow \gamma \gamma)}, \\
\frac{\mathrm{BR}\left(h \rightarrow \mu^{+} \mu^{-}\right)}{\mathrm{BR}(h \rightarrow \gamma \gamma)} . &
\end{array}
$$

These ratios all involve rare decay modes in which the Higgs can be reconstructed as a resonance, so they can be detected in the major production mode $g g \rightarrow h$ at low Higgs boson $p_{T}$. The ATLAS Collaboration has estimated that the first of these ratios can be measured to $3.6 \%$ accuracy in the full LHC program with $3000 \mathrm{fb}^{-1}$ [52]. We believe that, with an analysis specifically designed to cancel systematic errors, it will be possible to reach the statistics-limited accuracy of $2 \%$. This means that the combination of $c_{I}$ coefficients in $\zeta_{A}$ will be constrained to $10^{-4}$ accuracy. For the more difficult decays to $Z \gamma$ and $\mu^{+} \mu^{-}$, ATLAS has estimated eventual accuracies in these ratios of $31 \%$ and $12 \%$, respectively [52,53]. These measurements can be converted to partial width measurements when the absolute partial width $\Gamma\left(h \rightarrow Z Z^{*}\right)$ is measured at future $e^{+} e^{-}$colliders.

A $C P$-violating contribution to the $h \rightarrow \gamma \gamma$ decay from the operators in Eq. (2) would give a strictly additive contribution to the total rate of $h \rightarrow \gamma \gamma$ decay. A constraint of $2 \%$ on deviations from the SM in $\Gamma(h \rightarrow \gamma \gamma)$ would then place a constraint on the $\tilde{c}_{B B}$ coefficient in Eq. (2) at about $1 \%$. This is a strong enough constraint that this $C P$ violating coefficient can be ignored in our analysis.

\section{CONSTRAINTS FROM $e^{+} e^{-} \rightarrow Z h$}

At this point, all of the original 13 parameters are strongly constrained except for $c_{W W}$ and the parameter $c_{H}$ that appears only in Higgs decays. In this section, we explain how to determine them through the study of the process $e^{+} e^{-} \rightarrow Z h$. Our results for the total cross section in $e^{+} e^{-} \rightarrow Z h$ are similar to those in Ref. [5], but we also consider other observables of this process.

It is important to recall here that the parameter $c_{H}$ appears in the normalization of all Higgs couplings through the field-strength renormalization (8). Thus, it is not possible to determine $c_{H}$ unambiguously without measuring an absolute Higgs production or decay rate. Measurements of $\sigma \cdot B R$ are not sufficient. The total cross section for $e^{+} e^{-} \rightarrow Z h$ can be measured by tagging a $Z$ boson at the correct energy to be recoiling against a Higgs boson without the need for any information from the Higgs decay products. Thus, in principle, it provides a way to measure $c_{H}$. In the EFT formalism, there are complications from the fact that other EFT parameters also affect the size of the cross section. We will discuss the untangling of this parameter dependence at the end of this section and again in Sec. VIII.

The amplitudes for the reaction $e^{+} e^{-} \rightarrow Z h$ have a very simple form. For each initial polarization state $e_{L}^{-} e_{R}^{+}$or $e_{R}^{-} e_{L}^{+}$, there are three helicity amplitudes, corresponding to the three possible $Z$ boson polarization states. However, the two amplitudes with transverse $Z$ polarizations are related by $C P$, so there are only two independent amplitudes. Further, at the tree level within the EFT description, at a fixed c.m. energy, these amplitudes can be written with only two free parameters.

To describe these amplitudes, it is most convenient to begin by considering only the contribution from $s$-channel $Z$ boson exchange using the very simple-apparently, oversimplified-phenomenological Lagrangian

$$
\Delta \mathcal{L}=\frac{m_{Z}^{2}}{v}(1+a) h Z_{\mu} Z^{\mu}+\frac{1}{2} \frac{b}{v} h Z_{\mu \nu} Z^{\mu \nu} .
$$

Let $E_{Z}$ and $k$ be the energy and momentum of the $Z$ in the c.m. frame. Then we find that, for $e_{L}^{-} e_{R}^{+}$, this gives the helicity amplitudes 


$$
\begin{aligned}
& i \mathcal{M}\left(e^{+} e^{-} \rightarrow Z( \pm 1) h\right) \\
& =i g_{L} \frac{\sqrt{2 s}}{\left(s-m_{Z}^{2}\right)}\left[(1+a) \frac{m_{Z}^{2}}{v}+b \frac{E_{Z} \sqrt{s}}{v}\right](\cos \theta \pm 1), \\
& i \mathcal{M}\left(e^{+} e^{-} \rightarrow Z(0) h\right) \\
& =i g_{L} \frac{\sqrt{2 s}}{\left(s-m_{Z}^{2}\right)}\left[(1+a) \frac{m_{Z} E_{Z}}{v}+b \frac{m_{Z} \sqrt{s}}{v}\right](\sqrt{2} \sin \theta),
\end{aligned}
$$

where $\theta$ is the polar angle in production and the amplitudes are labeled by the $Z$ helicity. For $e_{R}^{-} e_{L}^{+}$, the helicity amplitudes take the same form except for the substitution of $g_{R}$ for $g_{L}$ and $(\cos \theta \mp 1)$ for $(\cos \theta \pm 1)$. These helicity amplitudes control the total cross section, the $Z h$ angular distributions, and the distributions of the $Z$ decay angle. In particular, the total cross section for a polarized initial state is given for $e_{L}^{-} e_{R}^{+}$by

$$
\begin{aligned}
\sigma\left(e_{L}^{-} e_{R}^{+} \rightarrow Z h\right) \\
=\frac{1}{6 \pi} \frac{g^{2}}{c_{w}^{2}} \frac{m_{Z}^{4} / v^{2}}{\left(s-m_{Z}^{2}\right)^{2}} \cdot \frac{2 k}{\sqrt{s}} \cdot\left(2+\frac{E_{Z}^{2}}{m_{Z}^{2}}\right) \\
\quad \cdot\left(\frac{1}{2}-s_{w}^{2}\right)^{2}\left((1+2 a)+6 b \frac{E_{Z} \sqrt{s}}{m_{Z}^{2}\left(2+E_{Z}^{2} / m_{Z}^{2}\right)}\right) .
\end{aligned}
$$

For $e_{R}^{-} e_{L}^{+}$, we have the same expression with the substitution $\left(\frac{1}{2}-s_{w}\right)^{2} \rightarrow s_{w}^{2}$.

In Ref. [54], it was shown how to obtain the values of the parameters $a$ and $b$ by fitting to the production and decay angular distributions in $e^{+} e^{-} \rightarrow Z h$ events. Using a full simulation with the ILD detector model and the $4 \mathrm{ab}^{-1}$ event sample expected for the ILC at $500 \mathrm{GeV}$, it was shown that the parameters $a$ and $b$ can be constrained at the percent level. The precise uncertainties expected, including their correlation, are given in Appendix B. To the accuracy of the study, these uncertainties are independent of the initial $e^{+} e^{-}$polarization state.

We can connect this analysis to the EFT parametrization of new physics effects by noting that the complete tree-level calculation of the helicity amplitudes for $e^{+} e^{-} \rightarrow Z h$ gives results that are still of the form of Eq. (40) for appropriate identification of the parameters $a$ and $b$. The complete set of Feynman diagrams is shown in Fig. 4. This includes a diagram with $s$-channel $Z$ exchange (with the $s$-channel $A Z$ mixing already included in the expressions for $g_{L}$ and $g_{R}$ ), a diagram with $s$-channel photon exchange that makes use of the $\zeta_{A Z}$ vertex, and a contact interaction proportional to $\left(c_{H L}+c_{H L}^{\prime}\right)$ or $c_{H E}$. Diagrams with $A Z$ kinetic mixing on the final-state line are of order $c_{I}^{2}$ and so are not included in our calculation.

Evaluating the diagrams in Fig. 4 and also expanding the SM dependence of the prefactors, we find, for $e_{L}^{-} e_{R}^{+}$,

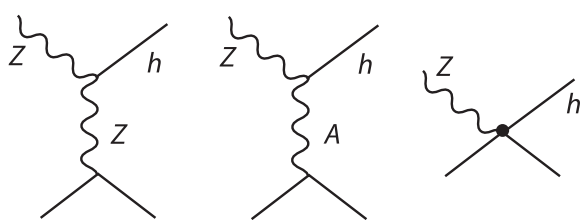

FIG. 4. Feynman diagrams contributing to the amplitudes for $e^{+} e^{-} \rightarrow Z h$.

$$
\begin{aligned}
a_{L}= & \delta g_{L}+2 \delta m_{Z}-\delta v+\eta_{Z}+\frac{\left(s-m_{Z}^{2}\right)}{2 m_{Z}^{2}\left(1 / 2-s_{w}^{2}\right)}\left(c_{H L}+c_{H L}^{\prime}\right) \\
& +k_{Z} \delta m_{Z}+k_{h} \delta m_{h}, \\
b_{L}= & \zeta_{Z}+\frac{s_{w} c_{w}}{\left(1 / 2-s_{w}^{2}\right)} \frac{\left(s-m_{Z}^{2}\right)}{s} \zeta_{A Z} .
\end{aligned}
$$

Similarly, for $e_{R}^{-} e_{L}^{+}$,

$$
\begin{aligned}
a_{R}= & \delta g_{R}+2 \delta m_{Z}-\delta v+\eta_{Z} \\
& -\frac{\left(s-m_{Z}^{2}\right)}{2 m_{Z}^{2}\left(s_{w}^{2}\right)} c_{H E}+k_{Z} \delta m_{Z}+k_{h} \delta m_{h}, \\
b_{R}= & \zeta_{Z}-\frac{c_{w}}{s_{w}} \frac{\left(s-m_{Z}^{2}\right)}{s} \zeta_{A Z} .
\end{aligned}
$$

The expressions for $a_{L}$ and $a_{R}$ include the kinematic factors

$k_{Z} \delta m_{Z}+k_{h} \delta m_{h}=\frac{1}{2} \delta\left[\frac{1}{\left(s-m_{Z}^{2}\right)^{2}} \cdot \frac{k}{\sqrt{s}} \cdot\left(2+\frac{E_{Z}^{2}}{m_{Z}^{2}}\right)\right]$.

The expansions of these expressions in terms of the $c_{I}$ are given in Appendix A. Note, in particular, that, up to parameters that have already been constrained as explained in the previous sections, $\eta_{Z}=-\frac{1}{2} c_{H}$ and $\zeta_{Z}=c_{w}^{2}\left(8 c_{W W}\right)$. Then, in principle, the percent-level constraints on the $a$ and $b$ coefficients will become percent-level constraints on the parameters $c_{H}$ and $c_{W W}$. At this point, we have put constraints on all of the EFT parameters that contribute to the cross section for $e^{+} e^{-} \rightarrow Z h h$ except for the parameter $c_{6}$ that we hope to determine from this reaction.

Table II shows the $1 \sigma$ errors on the EFT parameters obtained from the various stages of our fit. The first four columns of the table show the results from the fits described up to this point. The fits have increasing numbers of parameters, from seven parameters in the precision electroweak fit to 22 parameters in the full ILC fit. In each fit, we set the parameters not yet included to zero. The analysis of this paper concentrates on $500 \mathrm{GeV}$ measurements, but we also show for reference the fit results for $250 \mathrm{GeV}$ measurements. The table shows the progression that we have explained in this paper: precision electroweak fir fixes three EFT coefficients, taken here to be $c_{T}, c_{H E}$, and $c_{H L}$, to below the $10^{-3}$ level. The measurement of $e^{+} e^{-} \rightarrow W^{+} W^{-}$ adds constraints on $c_{H L}^{\prime}$ and $8 c_{W B}$. The LHC measurements 
TABLE II. $1 \sigma$ uncertainties, in $\%$, on EFT coefficients at different stages of this analysis. As more information is included, more parameters can be added to the fit. Parameters that are not yet included are set to 0 and marked in the table with.. . First column: Precision electroweak only (seven-parameter fit). Second column: Add $e^{+} e^{-} \rightarrow W W$ (ten-parameter fit). Third column: Add LHC measurements (12-parameter fit). Fourth column: Add the $e^{+} e^{-} \rightarrow Z h$ cross section, angular distribution, and polarization asymmetry (13-parameter fit). Fifth column: Add $e^{+} e^{-} \rightarrow \nu \bar{\nu} h$ and all $\sigma \cdot B R$ measurements, as described in Sec. VII and in Ref. [23] (22-parameter fit). In the top half of the table, the $e^{+} e^{-}$data is the expectation for $2000 \mathrm{fb}^{-1}$ at $250 \mathrm{GeV}$. In the bottom half of the table, the $e^{+} e^{-}$data is the expectation for $4000 \mathrm{fb}^{-1}$ at $500 \mathrm{GeV}$. The last column in the bottom half shows the result from the full ILC program at 250 and $500 \mathrm{GeV}$.

\begin{tabular}{lccccc}
\hline \hline \multicolumn{5}{c}{$250 \mathrm{GeV}$} \\
\hline$c_{I}$ & Prec. EW & $+W W$ & + LHC & + Zh & ILC 250 \\
\hline$c_{T}$ & 0.011 & 0.051 & 0.051 & 0.048 & 0.052 \\
$c_{H E}$ & 0.043 & 0.026 & 0.085 & 0.047 & 0.055 \\
$c_{H L}$ & 0.042 & 0.035 & 0.035 & 0.032 & 0.039 \\
$c_{H L}^{\prime}$ & $\ldots$ & 0.028 & 0.028 & 0.028 & 0.047 \\
$8 c_{W B}$ & $\ldots$ & 0.078 & 0.080 & 0.076 & 0.090 \\
$8 c_{B B}$ & $\ldots$ & $\ldots$ & 0.20 & 0.16 & 0.11 \\
$8 c_{W W}$ & $\ldots$ & $\ldots$ & 0.21 & 0.13 & 0.13 \\
$c_{H}$ & $\ldots$ & $\ldots$ & $\ldots$ & 1.12 & 1.20 \\
\hline \hline
\end{tabular}

$500 \mathrm{GeV}$

\begin{tabular}{lcccccc}
\hline$c_{I}$ & Prec. EW & $+W W$ & $+\mathrm{LHC}$ & $+Z h$ & ILC 500 & $250+500$ \\
\hline$c_{T}$ & 0.011 & 0.046 & 0.047 & 0.041 & 0.037 & 0.030 \\
$c_{H E}$ & 0.043 & 0.015 & 0.077 & 0.040 & 0.010 & 0.009 \\
$c_{H L}$ & 0.042 & 0.030 & 0.030 & 0.027 & 0.016 & 0.013 \\
$c_{H L}^{\prime}$ & $\ldots$ & 0.027 & 0.028 & 0.026 & 0.014 & 0.011 \\
$8 c_{W B}$ & $\ldots$ & 0.070 & 0.072 & 0.067 & 0.052 & 0.041 \\
$8 c_{B B}$ & $\ldots$ & $\ldots$ & 0.20 & 0.15 & 0.088 & 0.062 \\
$8 c_{W W}$ & $\ldots$ & $\ldots$ & 0.21 & 0.11 & 0.044 & 0.039 \\
$c_{H}$ & $\ldots$ & $\ldots$ & $\ldots$ & 4.78 & 1.24 & 0.65 \\
\hline \hline
\end{tabular}

of ratios of branching ratios constrain two additional linear combinations of the dimension-six terms involving squares of field strengths and thus provide significant constraints on $8 c_{B B}$ and $8 c_{W W}$. Finally, adding information from $e^{+} e^{-} \rightarrow$ $Z h$ sharpens all of these constraints while also constraining the coefficient $c_{H}$.

These constraints, however, are not yet sufficiently powerful to achieve our goal in this paper. The problem comes from the fact that, although the errors on $c_{H E}, c_{H L}$, and $c_{H L}^{\prime}$ are quite small, these parameters appear in Eqs. (42) and (43) with very large coefficients, of order $2 s / m_{Z}^{2} \sim 60$. This limits the power of these equations to constrain $c_{H}$. The uncertainty on $c_{H}$ resulting from the analysis described so far is about $5 \%$, as shown in the first entry in the last line of Table II. This is already consistent with our approximation of ignoring terms of other $c_{H}^{2}$ and other terms quadratic in the EFT coefficients. However, this constraint is weaker than what we need for the determination of the triple Higgs coupling. The constraint on $c_{W W}$, which comes from the angular distribution and polarization asymmetry in $e^{+} e^{-} \rightarrow Z h$, is already quite strong.

It should be noted that a similar analysis at $250 \mathrm{GeV}$, where the coefficients of the contact terms are smaller by a factor of 4 , gives a much stronger constraint on $c_{H}$. This is shown in the first entry in the last line of the top half of Table II. One can see from the sixth column in the bottom half of Table II that a combined analysis of 250 and $500 \mathrm{GeV}$ data is especially powerful to constrain the effects of the contact interactions.

In any event, it is also possible to improve the constraint on the parameter $c_{H}$ by including additional information from $e^{+} e^{-}$Higgs reactions. In Secs. VI and VII, we will explain how to improve our fit using information from the $W$ fusion reaction $e^{+} e^{-} \rightarrow \nu \bar{\nu} h$ and from the Higgs decay partial widths. After we add this information, the fit results will evolve further to those shown in the fifth and sixth columns of Table II.

The analysis in Ref. [54] also considered the addition of a third, $C P$-violating, term in the effective Lagrangian,

$$
\Delta \mathcal{L}=\frac{1}{2} \frac{\tilde{b}}{v} h Z_{\mu \nu} \tilde{Z}^{\mu \nu}
$$

It was found that the same data set constrains the coefficient $\tilde{b}$ to be less than $1 \%$. This is the final piece of information that we need to demonstrate that-if significant $C P$ violating terms are not actually generated by new physics-the possibility of $C P$-violating operators does not affect the uncertainties estimated in our analysis.

\section{CONSTRAINTS FROM $e^{+} e^{-} \rightarrow \nu \bar{\nu} h$}

To obtain additional constraints on $c_{H}$, we now turn to the process $e^{+} e^{-} \rightarrow \nu \bar{\nu} h$. Unlike $e^{+} e^{-} \rightarrow Z h$, it is not possible to measure this total cross section directly. But still, this process plays an important role in the extraction of Higgs boson partial widths from $e^{+} e^{-}$data.

The Feynman diagrams for $e^{+} e^{-} \rightarrow \nu \bar{\nu} h$ are shown in Fig. 5. There is one helicity amplitude, for $e_{L}^{-} e_{R}^{+} \rightarrow \nu_{L} \bar{\nu}_{R} h$. The first diagram is the one that appears at tree level in the SM. The additional three diagrams involve contact interactions proportional to $c_{H L}$ and $c_{H L}^{\prime}$. There are further contributions from the process $e^{+} e^{-} \rightarrow Z h, Z \rightarrow \nu \bar{\nu}$, but

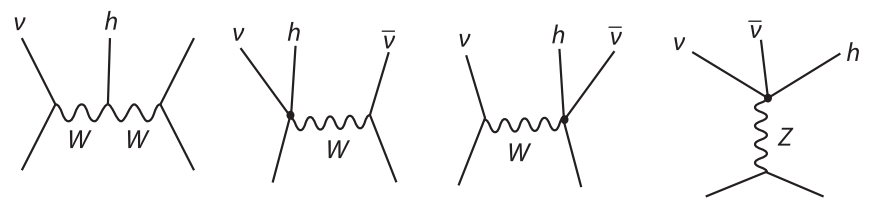

FIG. 5. Feynman diagrams contributing to the amplitudes for $e^{+} e^{-} \rightarrow \nu \bar{\nu} h$. 
these are important only when the final $Z$ is close to its mass shell. We will ignore them, and, more generally, we will ignore interference between the $W$ fusion reaction and the $e^{+} e^{-} \rightarrow Z h$ reaction.

The first diagram shown in Fig. 5 has the value

$$
\begin{aligned}
i \mathcal{M}= & i \frac{g_{W}^{2}}{2}\left\{\frac{2 m_{W}^{2}}{v}\left(1+\eta_{W}\right) g^{\mu \nu}-\frac{2}{v} \zeta_{W}\left(q_{1} \cdot q_{2} g^{\mu \nu}-q_{1}^{\nu} q_{2}^{\mu}\right)\right\} \\
& \cdot \frac{1}{\left(q_{1}^{2}-m_{W}^{2}\right)\left(q_{2}^{2}-m_{W}^{2}\right)} \\
& \cdot \bar{u}_{L}(\nu) \gamma_{\mu} u_{L}\left(e^{-}\right) \bar{v}_{R}\left(e^{+}\right) \gamma_{\nu} v_{R}(\bar{\nu})
\end{aligned}
$$

where $q_{1}, q_{2}$ are the momenta of the two off-shell $W$ bosons. Including also the various contact interactions, the full expression for this amplitude is

$$
\begin{aligned}
i \mathcal{M}= & i \frac{g_{W}^{2}}{2}\left\{\frac{2 m_{W}^{2}}{v}\left(1+\eta_{W}\right) g^{\mu \nu}-\frac{2}{v} \zeta_{W}\left(q_{1} \cdot q_{2} g^{\mu \nu}-q_{1}^{\nu} q_{2}^{\mu}\right)\right. \\
& \left.+2 c_{H L}^{\prime}\left(\frac{q_{1}^{2}-m_{W}^{2}+q_{2}^{2}-m_{W}^{2}}{2 m_{W}^{2}}\right)\right\} \cdot \frac{1}{\left(q_{1}^{2}-m_{W}^{2}\right)\left(q_{2}^{2}-m_{W}^{2}\right)} \\
& \cdot \bar{u}_{L}(\nu) \gamma_{\mu} u_{L}\left(e^{-}\right) \bar{v}_{R}\left(e^{+}\right) \gamma_{\nu} v_{R}(\bar{\nu})-\frac{g^{2}}{c_{w}^{2}} \frac{g^{\mu \nu}}{v\left(s-m_{Z}^{2}\right)} \\
& \left(c_{H L}-c_{H L}^{\prime}\right) \cdot \bar{u}_{L}(\nu) \gamma_{\mu} v_{R}(\bar{\nu}) \bar{v}_{R}\left(e^{+}\right) \gamma_{\nu} v_{R}(\bar{\nu}) .
\end{aligned}
$$

It is not straightforward to quote analytic results for the dependence of the total cross section on the EFT parameters. However, we can integrate the expression (47) over the three-body phase space numerically to compute the fully polarized cross section. We obtain, for $\sqrt{s}=250 \mathrm{GeV}$,

$$
\begin{aligned}
\sigma /(S M)= & 1+2 \eta_{W}-2 \delta v+2 \delta g_{W}-1.6 \delta m_{W}-3.7 \delta m_{h} \\
& -0.22 \zeta_{W}-6.4 c_{H L}^{\prime}-0.37\left(c_{H L}-c_{H L}^{\prime}\right),
\end{aligned}
$$

for $\sqrt{s}=350 \mathrm{GeV}$,

$$
\begin{aligned}
\sigma /(S M)= & 1+2 \eta_{W}-2 \delta v+2 \delta g_{W}-1.2 \delta m_{W}-2.0 \delta m_{h} \\
& -0.32 \zeta_{W}-7.5 c_{H L}^{\prime}-0.28\left(c_{H L}-c_{H L}^{\prime}\right),
\end{aligned}
$$

for $\sqrt{s}=380 \mathrm{GeV}$,

$$
\begin{aligned}
\sigma /(S M)= & 1+2 \eta_{W}-2 \delta v+2 \delta g_{W}-1.1 \delta m_{W}-1.7 \delta m_{h} \\
& -0.34 \zeta_{W}-7.8 c_{H L}^{\prime}-0.26\left(c_{H L}-c_{H L}^{\prime}\right),
\end{aligned}
$$

and for $\sqrt{s}=500 \mathrm{GeV}$,

$$
\begin{aligned}
\sigma /(S M)= & 1+2 \eta_{W}-2 \delta v+2 \delta g_{W}-0.85 \delta m_{W}-1.2 \delta m_{h} \\
& -0.39 \zeta_{W}-8.8 c_{H L}^{\prime}-0.19\left(c_{H L}-c_{H L}^{\prime}\right) .
\end{aligned}
$$

Each expression contains $\left(-c_{H}-a\left(8 c_{W W}\right)\right)$, with the first term coming from $\eta_{W}$ and the second from $\zeta_{W}$. The coefficient $a$ of $\zeta_{W}$ increases slowly with center-of-mass energy. Thus, measurements of $\sigma \cdot B R$ for $W W$ fusion to a Higgs boson and then to a given final state can constrain the parameters $c_{H}$ and $c_{W W}$ in the context of a global fit to Higgs boson data.

In the second line of each of these expressions, the second term comes from the diagrams with contact interactions and $t$-channel $W$ exchange. The numerical coefficients in these $c_{H L}^{\prime}$ terms are large and increase with center-of-mass energy, just as we saw for the contact contributions in $e^{+} e^{-} \rightarrow Z h$. However, now there is an interesting possibility. If the cross sections for both processes are measured, the contact interaction coefficients are overdetermined and can be constrained even more strongly than they are from precision electroweak data. We will see in Sec. VIII that this is indeed the case.

Since the total cross section for $e^{+} e^{-} \rightarrow \nu \bar{\nu} h$ cannot be measured directly, we must consider the formulas (48), (49), and (51) in conjunction with formulas for Higgs decay processes. We develop these formulas in the next section.

\section{EFT FORMALISM FOR GENERAL HIGGS BOSON COUPLINGS}

In the process of answering the main issue of this paper, we have already come very close to assembling the complete set of formulas that we need to represent general Higgs boson cross sections at $e^{+} e^{-}$colliders in terms of EFT coefficients. In this section, we derive the remaining formulas needed for such an analysis. These are the formulas for the various Higgs decay widths. The implications of the formalism of this paper for the extraction of Higgs couplings at $e^{+} e^{-}$colliders will be discussed in a companion paper [23].

In Sec. IV, we derived expansions for two of the minor decay amplitudes, $h \rightarrow \gamma \gamma$ and $h \rightarrow Z \gamma$. What remains is to derive formulas for the major Higgs boson decay amplitudes to fermions, $W W^{*}$, and $Z Z^{*}$.

\section{A. Higgs decay to fermions and gluons}

At the level of this tree-level analysis, the appropriate treatment of Higgs decays to fermions is very simple. For definiteness, consider the case of $h \rightarrow \tau^{+} \tau^{-}$. Deviations in the Higgs couplings from the SM expectation are generated by the dimension-six operator

$$
\Delta \mathcal{L}=-c_{\tau \Phi} \frac{y_{\tau}}{v^{2}}\left(\Phi^{\dagger} \Phi\right) \bar{L}_{3} \cdot \Phi \tau_{R}+\text { H.c. },
$$

where $y_{\tau}$ is the bare Yukawa coupling. Then

$$
m_{\tau}=\frac{y_{\tau} v}{\sqrt{2}}\left(1+\frac{1}{2} c_{\tau \Phi}\right) .
$$

Substituting $m_{\tau}$ for $y_{\tau}$ using this formula and including the Higgs field-strength renormalization from Eq. (8), the $\tau$ couplings to the Higgs boson becomes 


$$
\Delta \mathcal{L}=-m_{\tau} \bar{\tau} \tau \cdot\left(1-\frac{1}{2} c_{H}+c_{\tau \Phi}\right) \cdot \frac{h}{v}
$$

The variation of the Higgs width is then

$$
\delta \Gamma\left(h \rightarrow \tau^{+} \tau^{-}\right)=1-c_{H}+2 c_{\tau \Phi}+\delta,
$$

where $\delta=2 \delta m_{\tau}+\delta m_{h}-2 \delta v$. For simplicity, we will absorb this term into $c_{\tau \Phi}$.

A similar logic applies to the Higgs boson couplings to $b, c, \mu$, and other fermions. Then, we will write

$$
\begin{aligned}
\delta \Gamma(h \rightarrow b \bar{b}) & =1-c_{H}+2 c_{b \Phi}, \\
\delta \Gamma(h \rightarrow c \bar{c}) & =1-c_{H}+2 c_{c \Phi}, \\
\delta \Gamma\left(h \rightarrow \tau^{+} \tau^{-}\right) & =1-c_{H}+2 c_{\tau \Phi}, \\
\delta \Gamma\left(h \rightarrow \mu^{+} \mu^{-}\right) & =1-c_{H}+2 c_{\mu \Phi} .
\end{aligned}
$$

QCD corrections provide factors that commute with the effect of dimension-six operators and so do not affect these formulas. Mixed QCD-electroweak corrections will give loop-level corrections to these formulas.

The effect of dimension-six operators on the partial width for $h \rightarrow g g$ is more complex. The first contribution to this width in the SM comes at the loop level. Dimension-six operators correct this expression through a tree-level contribution proportional to the coefficient $c_{G G}$ of a gluonic operator similar to that for $c_{W W}$, and through corrections to the SM loop diagrams, for example, from $c_{t \Phi}$. Fortunately, for an on-shell Higgs boson, it is a good approximation to summarize all of these effects as an effective coupling of the form

$$
\delta \mathcal{L}=\mathcal{A} \frac{h}{v} G_{\mu \nu} G^{\mu \nu}
$$

In fitting Higgs couplings, we will write

$$
\delta \Gamma(h \rightarrow g g)=1-c_{H}+2 c_{g \Phi},
$$

letting the parameter $c_{g \phi}$ stand in for all of the effects just described.

A full description of the $h \rightarrow g g$ width in the EFT formalism would include the dependence of this partial width on the canonical EFT parameters $c_{G G}, c_{t \Phi}$, and $c_{t G}$ [27], with small corrections from other dimension-six operators. That discussion is beyond the scope of this paper. The leading effects can be disentangled by measurements of Higgs emission from $t \bar{t}$, Higgs production in $p p$ collisions at high $p_{T}$, and top quark pair production at high energy. A part of this analysis was given in Refs. [55,56].

\section{B. Higgs decay to $W W^{*}$ and $Z Z^{*}$}

The Higgs decay widths to $W W^{*}$ and $Z Z^{*}$ also bring in new EFT vertices. However, in this case, the new terms can be constrained by additional precision electroweak measurements.

As a first step in this analysis, consider a model of $h \rightarrow$ $W W^{*}$ in which the $W^{-}$converts only to $e^{-} \bar{\nu}$ and the $W^{+}$ converts only to $e^{+} \nu$. In this case, the $W$ width would be

$$
\begin{aligned}
\Gamma_{W, \text { simple }} & =\frac{g_{W}^{2} m_{W}}{48 \pi} \\
& =\frac{g^{2} m_{W}}{48 \pi}\left(1+2 \delta g+\delta m_{W}+2 c_{H L}^{\prime}+\delta Z_{W}\right) .
\end{aligned}
$$

For an off-shell $W$, we will use the propagator

$$
D(q)=1 /\left(q^{2}-m_{W}^{2}+i q^{2}\left(\Gamma_{W} / m_{W}\right)\right)
$$

with a $q^{2}$-dependent width.

It is straightforward to compute the rate of the $h \rightarrow W W^{*}$ decay in this model. The Feynman diagrams are shown in Fig. 6. Note that, in addition to the usual SM diagram, there are contributions from the contact interaction proportional to $c_{H L}^{\prime}$. The decay amplitude is

$$
\begin{aligned}
i \mathcal{M}= & i \frac{g_{W}^{2}}{2}\left\{\frac { 2 m _ { W } ^ { 2 } } { v } g ^ { \mu \nu } \left[\left(1+\eta_{W}\right) D\left(q_{1}^{2}\right) D\left(q_{2}^{2}\right)\right.\right. \\
& \left.+\frac{c_{H L}^{\prime}}{2 m_{W}^{2}}\left(D\left(q_{1}^{2}\right)+D\left(q_{2}^{2}\right)\right)\right] \\
& \left.-\frac{2}{v} \zeta_{W}\left(q_{1}^{2} \cdot q_{2}^{2} g^{\mu \nu}-q_{1}^{\nu} q_{2}^{\mu}\right)\right\} \\
& \times \bar{u}_{L}\left(e^{-}\right) \gamma_{\mu} v_{R}(\bar{\nu}) \bar{u}_{L}(\nu) \gamma_{\nu} v_{R}\left(e^{+}\right),
\end{aligned}
$$

where $q_{1}, q_{2}$ are the momenta of the $W^{-}$and $W^{+}$. Integrating this expression over phase space and using Eq. (59) to simplify the numerator, we find

$$
\begin{aligned}
\Gamma /(S M)= & 1+2 \eta_{W}-2 \delta v-11.7 \delta m_{W}+13.6 \delta m_{h} \\
& -0.75 \zeta_{W}-0.88 C_{W}+1.06 \delta \Gamma_{W},
\end{aligned}
$$

where we have written $C_{W}=c_{H L}^{\prime}$. There is a partial cancellation between the factors of $c_{H L}^{\prime}$ that appear
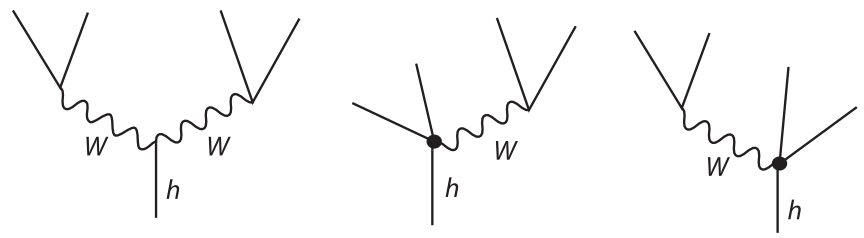

FIG. 6. Feynman diagrams contributing to the amplitudes for $h \rightarrow W W^{*}$. 
explicitly due to the contact interactions and the factors that appear in $\Gamma_{W}$ through Eq. (59).

In reality, the $W$ boson can decay to all of the SM $S U(2)$ doublets except $(t, b)$. This brings in additional $c_{H X}^{\prime}$ coefficients for the first and second quark generations. Fortunately, these new coefficients appear only in the same combination that appears in the full $W$ width. Let

$$
C_{W}=\sum_{X} c_{X}^{\prime} \mathcal{N}_{X} / \sum_{X} \mathcal{N}_{X}
$$

where $X$ runs over the five SM doublets that appear in $W$ decays, $c_{X}^{\prime}$ is the coefficient of the operator similar to that multiplying $c_{H L}^{\prime}$, and $\mathcal{N}_{X}$ is the number of color states for that doublet, including the QCD radiative correction. Then, including all first-order EFT corrections, the $W$ width is given by

$\Gamma_{W}=\frac{g^{2} m_{W}}{48 \pi}\left(\sum_{X} \mathcal{N}_{X}\right) \cdot\left(1+2 \delta g+\delta m_{W}+\delta Z_{W}+2 C_{W}\right)$.

The expression (62) remains valid, but with $c_{H L}^{\prime}$ replaced by $C_{W}$. We can constrain the value of $C_{W}$ by a measurement of the $W$ total width, and then Eq. (62) becomes an additional constraint on the EFT parameters $c_{H}$ and $c_{W W}$.

It is also striking that the expression (62) shows a very strong dependence on the masses of the $W$ boson and the Higgs boson. The improvements in these quantities expected from the LHC and ILC and listed in Table I will be important to make use of the Higgs boson width to $W W^{*}$ in a global fit to the Higgs boson couplings.

The analysis of $h \rightarrow Z Z^{*}$ is formally quite similar, but there is some additional bookkeeping to do. We write the SM coupling of one chiral flavor $X$ to the $Z$ boson as

$$
\Delta \mathcal{L}=\frac{g}{c_{w}} Q_{Z X} Z_{\mu} \bar{X} \gamma^{\mu} X
$$

where $Q_{Z X}=I_{X}^{3}-s_{w}^{2} Q_{X}$, where $I_{X}^{3}$ and $Q_{X}$ are the weak isospin and the electric charge of $X$. The contact interactions yield an additional direct coupling

$$
\Delta \mathcal{L}=\frac{g}{c_{w}} c_{X} Z_{\mu} \bar{X} \gamma^{\mu} X\left(1+2 \frac{h}{v}+\cdots\right),
$$

introducing a new parameter $c_{X}$ for each chiral flavor. When we include this effect and all other first-order EFT corrections, the coupling of the $Z$ to $X \bar{X}$ is modified to

$$
\begin{aligned}
g_{X}= & \frac{g}{c_{w}}\left[Q_{Z X}\left(1+c_{w}^{2} \delta g+s_{w}^{2} \delta g^{\prime}+\frac{1}{2} \delta Z_{Z}\right)\right. \\
& \left.+Q_{X}\left(2 s_{w}^{2} c_{w}^{2}\left(\delta g-\delta g^{\prime}\right)+s_{w} c_{w} \delta Z_{A Z}\right)+c_{X}\right] .
\end{aligned}
$$

Then the total $Z$ width becomes

$$
\begin{aligned}
\Gamma_{Z}= & \frac{g^{2} m_{Z}}{24 \pi c_{w}^{2}}\left(\sum_{X} Q_{Z X}^{2} \mathcal{N}_{X}\right) \\
& \cdot\left[\left(1+2 c_{w}^{2} \delta g+2 s_{w}^{2} \delta g^{\prime}+\delta m_{Z}+\delta Z_{Z}\right)\right. \\
& \left.+\frac{\sum_{X} Q_{Z X} Q_{X} \mathcal{N}_{X}}{\sum_{X} Q_{Z X}^{2} \mathcal{N}_{X}}\left(4 s_{w}^{2} c_{w}^{2}\left(\delta g-\delta g^{\prime}\right)+s_{w} c_{w} \delta Z_{A Z}\right)\right] \\
& \cdot\left(1+2 C_{Z}\right),
\end{aligned}
$$

where

$$
C_{Z}=\frac{\sum_{X} c_{X} Q_{Z X} \mathcal{N}_{X}}{\sum_{X} Q_{Z X}^{2} \mathcal{N}_{X}}
$$

For the $Z$ decaying to SM fermions,

$$
\sum_{X} Q_{Z X}^{2} \mathcal{N}_{X}=3.75, \quad \sum_{X} Q_{Z X} Q_{X} \mathcal{N}_{X}=1.99 .
$$

The contact interaction also affects the $h \rightarrow Z Z^{*}$ decay by adding additional contact diagrams similar to those in Fig. 6. We find

$$
\begin{aligned}
\Gamma /(S M)= & 1+2 \eta_{Z}-2 \delta v-13.8 \delta m_{W}+15.6 \delta m_{h} \\
& -0.50 \zeta_{Z}-1.02 C_{Z}+1.18 \delta \Gamma_{Z} .
\end{aligned}
$$

So, here again, there is an extra EFT parameter, but it can be controlled by measurements of the $Z$ total width.

The conclusions of this section are summarized in Appendix A.

\section{THE TOTAL CROSS SECTION FOR $e^{+} e^{-} \rightarrow Z h h$}

We are now ready to describe the derivation of the parameter $c_{6}$ from the value of the total cross section for

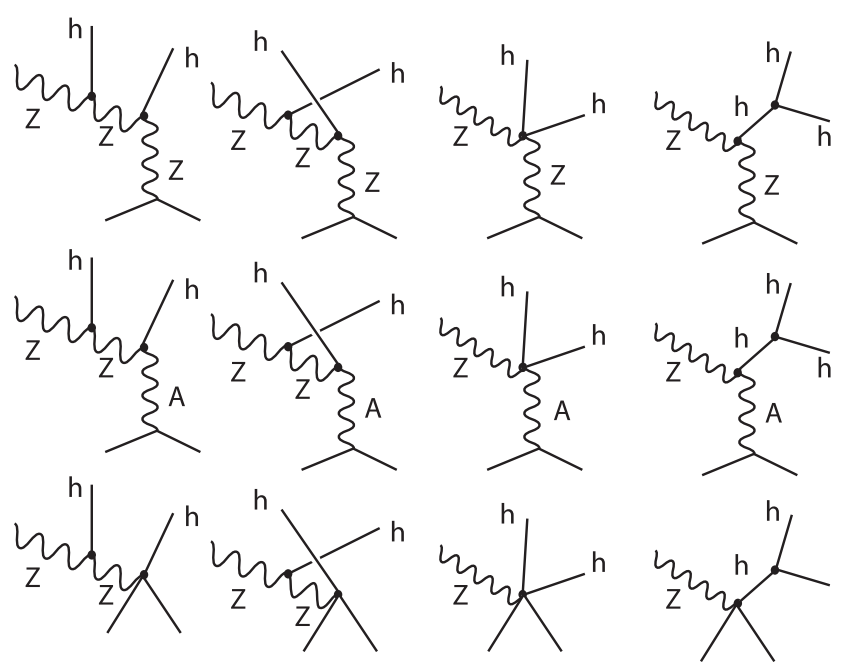

FIG. 7. Feynman diagrams contributing to the amplitudes for $e^{+} e^{-} \rightarrow Z h h$. 
$e^{+} e^{-} \rightarrow Z h h$. The tree-level Feynman diagrams for this process are shown in Fig. 7. Evaluating these diagrams and numerically integrating over three-body phase space, we will obtain an expression of a form similar to our cross section formulas for $e^{+} e^{-} \rightarrow \nu \bar{\nu} h$.

The diagrams in the first row of Fig. 7 are those of the Standard Model. However, in our EFT formalism, all $Z Z h$ vertices also include the renormalization of all Higgs vertices by $\delta Z_{h}$ and new structures proportional to $\zeta_{Z}$. The last diagram in this row contains the modification of the triple Higgs coupling proportional to $c_{6}$ but also the additional vertex structure from the term proportional to $\theta_{h}$ in Eq. (25). The diagram in the second row makes use of the $\zeta_{A Z}$ term that converts $A$ to $Z$ while emitting one or more Higgs bosons. Recall that kinetic mixing between $A$ and $Z$ in the $s$-channel propagator has already been taken into account in the parameters $g_{L}, g_{R}$. Diagrams with kinetic mixing beyond the first vertex are of order $c_{I}^{2}$ and can be ignored. The diagrams in the third row involve the contact interactions proportional to $\left(c_{H L}-c_{H L}^{\prime}\right)$ and $c_{H E}$. In all, there are many opportunities for EFT coefficients other than $c_{6}$ to influence the value of this cross section.

The amplitude for $e^{+} e^{-} \rightarrow Z h h$ depends on the initial beam polarization and on the final polarization state of the $Z$. We compute the cross section at $\sqrt{s}=500 \mathrm{GeV}$ for definite choices of the initial beam polarization and summed over $Z$ helicities. For a fully polarized initial state $e_{L}^{-} e_{R}^{+}$, we find

$$
\begin{aligned}
\sigma /(S M)= & 1+2 \delta g_{L}+1.40 \eta_{Z}+1.02 \eta_{Z Z}+18.6 \zeta_{Z}+24.8 \zeta_{A Z} \\
& +0.56 \eta_{h}-1.58 \theta_{h}+108.3\left(c_{H L}+c_{H L}^{\prime}\right) \\
& -3.9 \delta m_{h}+3.5 \delta m_{Z} .
\end{aligned}
$$

For a fully polarized initial state $e_{R}^{-} e_{L}^{+}$, we find

$$
\begin{aligned}
\sigma /(S M)= & 1+2 \delta g_{R}+1.40 \eta_{Z}+1.02 \eta_{Z Z}+18.6 \zeta_{Z}-28.7 \zeta_{A Z} \\
& +0.56 \eta_{h}-1.58 \theta_{h}-125.5 c_{H E} \\
& -3.9 \delta m_{h}+3.5 \delta m_{Z} .
\end{aligned}
$$

For an unpolarized $e^{+} e^{-}$initial state, we find

$$
\begin{aligned}
\sigma /(S M)= & 1+1.15 \delta g_{L}+0.85 \delta g_{R}+1.40 \eta_{Z}+1.02 \eta_{Z Z} \\
& +18.6 \zeta_{Z}+2.0 \zeta_{A Z}+0.56 \eta_{h}-1.58 \theta_{h} \\
& +62.1\left(c_{H L}+c_{H L}^{\prime}\right)-53.5 c_{H E} \\
& -3.9 \delta m_{h}+3.5 \delta m_{Z}
\end{aligned}
$$

These equations are rewritten with some convenient rearrangements of terms in Appendix A.

We find the dependence on EFT parameters shown in this equation to be quite surprising. It is well known that the dependence of the $e^{+} e^{-} \rightarrow$ Zhh cross section on the triple Higgs coupling is weak. Here, that dependence appears in the coefficient of $\eta_{h}=c_{6}+\cdots$. The relation

$$
\sigma /(S M)=1+0.56 c_{6}+\cdots
$$

agrees with Ref. [12] and earlier studies. What is remarkable is that the dependence on other parameters is much larger. We might pay particular attention to the dependence on $c_{H}$ and $c_{W W}$, the two parameters that are only fixed by single Higgs production processes. The parameter $c_{H}$ appears in $\eta_{Z}, \eta_{Z Z}, \eta_{h}$, and $\theta_{h}$. The parameter $c_{W W}$ appears in $\zeta_{Z}$ and $\zeta_{A Z}$; we omit a further dependence from the independently constrained $\delta g_{L . R}$. The sum of these terms gives (in the unpolarized case)

$$
\sigma /(S M)=1-4.15 c_{H}+15.1\left(8 c_{W W}\right)+\cdots
$$

The coefficients here are an order of magnitude larger than that in Eq. (75). In addition, the parameters $\left(c_{H L}+\right.$ $\left.c_{H L}^{\prime}\right)$ and $c_{H E}$, which are constrained by precision electroweak measurements, have very large coefficients, reflecting an $s / m_{Z}^{2}$ enhancement of their contributions. We have seen this effect already in both of the single Higgs boson reactions considered earlier in this paper. It is clear that, without precise constraints on the EFT parameters from all of the sources that we have discussed in this paper, it is not possible to convincingly attribute a measured increase in the double Higgs production cross section to a shift in the triple Higgs coupling.

We can discuss this quantitatively using a fit to the EFT parameters aside from $c_{6}$ using the inputs in Table I, for precision electroweak data, the inputs listed in Appendix B for $W^{+} W^{-}$, and the measurement of the $a_{L, R}$ and $b_{L, R}$ parameters in $e^{+} e^{-} \rightarrow Z h$. This fit involves 13 parameters: the four SM parameters and the nine EFT coefficients introduced in Sec. II. The fit results for the relevant $c_{I}$ parameters have already been shown in Table II. This fit leads to the following values for the root-mean-square errors (in \%) on EFT coefficients:

\begin{tabular}{ll|ll}
$A$ & {$\left[\left\langle A^{2}\right\rangle\right]^{1 / 2}$} & $A$ & {$\left[\left\langle A^{2}\right\rangle\right]^{1 / 2}$} \\
\hline$c_{H}$ & 4.8 & $\left(c_{H L}+c_{H L}^{\prime}\right)$ & 0.048 \\
$\left(8 c_{W W}\right)$ & 0.11 & $c_{H E}$ & 0.040 \\
$\left(-4.15 c_{H}+15.1\left(8 c_{W W}\right)\right)$ & 21 & $62.1\left(c_{H L}+c_{H L}^{\prime}\right)-53.5 c_{H E}$ & 4.9
\end{tabular}


We find for the root-mean-square uncertainty in the complete right-hand side of Eq. (74), omitting the dependence on $c_{6}$

$$
\left[\left\langle(\delta \sigma)^{2}\right\rangle\right]^{1 / 2}=14 \% .
$$

This means that a measurement of $c_{6}$ from the cross section for $e^{+} e^{-} \rightarrow Z h h$ will be subject to a $28 \%$ systematic uncertainty from the uncertainties in the other EFT parameters. So the logic that we have described is in principle valid, but it leads to a very large uncertainty from other new physics effects in the determination of $c_{6}$.

We pointed out at the end of Sec. V that this problem can be solved by adding data from the various $\sigma \cdot B R$ measurements possible with $e^{+} e^{-} \rightarrow \nu \bar{\nu} h$, together with information from $\sigma \cdot B R$ measurements in $e^{+} e^{-} \rightarrow Z h$. Given the absolute measurement of the total cross section for $e^{+} e^{-} \rightarrow Z h$, these additional measurements fix the various new parameters that appear in the Higgs boson decay amplitudes. Using the fit to these parameters, we can bootstrap the measurement of the total cross section for $e^{+} e^{-} \rightarrow Z h$ into a determination of the total cross section for $e^{+} e^{-} \rightarrow \nu \bar{\nu} h$ and the absolute normalization of the partial widths $\Gamma\left(h \rightarrow W W^{*}\right)$ and $\Gamma\left(h \rightarrow Z Z^{*}\right)$. This gives an independent way to determine $c_{H}$. This method is applied in the fits presented in the fifth and sixth columns of Table II, and one can see from that table that it is effective. The full set of inputs to these fits, and the results for Higgs boson couplings and decay amplitudes, were described in detail in Ref. [23].

Using the fit described in the final column of Table II, including all cross sections and branching fractions that will be measured at the ILC at 250 and $500 \mathrm{GeV}$, the errors reported in Eq. (77) improve to

\begin{tabular}{ll|ll}
$A$ & {$\left[\left\langle A^{2}\right\rangle\right]^{1 / 2}$} & $A$ & {$\left[\left\langle A^{2}\right\rangle\right]^{1 / 2}$} \\
\hline$c_{H}$ & 0.65 & $\left(c_{H L}+c_{H L}^{\prime}\right)$ & 0.014 \\
$\left(8 c_{W W}\right)$ & 0.039 & $c_{H E}$ & 0.009 \\
$\left(-4.15 c_{H}+15.1\left(8 c_{W W}\right)\right)$ & 2.8 & $62.1\left(c_{H L}+c_{H L}^{\prime}\right)-53.5 c_{H E}$ & 0.85
\end{tabular}

and the uncertainty in $\delta \sigma$ becomes

$$
\left[\left\langle(\delta \sigma)^{2}\right\rangle\right]^{1 / 2}=2.4 \% .
$$

At this point, the effects of other EFT coefficients contribute only a $5 \%$ systematic error to the determination of the parameter $c_{6}$, and so this parameter can be determined from the measurement of the $e^{+} e^{-} \rightarrow Z h h$ cross section with high precision in a model-independent way.

As an aside, we note that the full fits to Higgs observables give quite an impressive improvement in the uncertainties in the parameters $c_{H E}, c_{H L}$, and $c_{H L}^{\prime}$ from the original precision electroweak determination. In precision electroweak observables, the $c_{H L}$ and related parameters alter the $W$ and $Z$ couplings with coefficients that are of order 1. In the EFT formalism, these same parameters appear as contact interactions in the Higgs reactions, with coefficients that are enhanced by factors of order $s / m_{Z}^{2}$. Then the sensitivity to these factors is much stronger. The EFT formalism implies that the measurement of Higgs reactions can provide more powerful tests of deviations of the predictions of precision electroweak analysis than precision electroweak measurements themselves.

\section{CONCLUSIONS}

In this paper, we have assembled a complete formalism, valid at the tree level and to linear order in the coefficients of dimension-six operators, describing the possible new physics perturbations of the Standard Model predictions for precision electroweak observables, $e^{+} e^{-} \rightarrow W^{+} W^{-}$, and Higgs boson production and decay reactions. This formalism requires a fit to 14 variables for the determination of the triple Higgs coupling and an additional seven variables for a general analysis of Higgs decays to Standard Model particles. However, it provides a completely modelindependent description of the effects of new physics that arises at mass scales much larger than the mass of the Higgs boson.

It is challenging to fit this large number of parameters with high precision and with systematic understanding of the constraints. However, future $e^{+} e^{-}$colliders will be up to this challenge. We have shown that the determination of the parameters can make use of all of the important advantages of $e^{+} e^{-}$experimentation: beam polarization, the visibility of all relevant decay channels, and the ability to measure over essentially all of phase space. It is already understood that these are powerful capabilities, but it is wonderful to see in this analysis how these powerful measurements interlock to provide a rich and secure basis from which to search for new effects.

The analysis that we have described is particularly important for the determination of the triple Higgs coupling. This fundamental quantity of the Standard Model is never seen in isolation. It is always studied as an interference effect, in combination with many other particle vertices. We might be able to measure a deviation that could plausibly arise from a shift of the triple Higgs 
coupling, but to understand definitely that this and not some other perturbation is the cause, an analysis of the type described in this paper is required.

It is difficult to imagine repeating the analysis presented here with data from hadron colliders only. The use of hadronic initial states brings in many more unknown coefficients of dimension-six operators, while offering fewer tools to discriminate between their effects. For the triple Higgs coupling, there is the additional complication that the leading double Higgs production process, $g g \rightarrow h h$, is loop level in the Standard Model, which adds another layer of complexity.

Thus, a future $e^{+} e^{-}$collider is not only sufficient but also essential for a full understanding of the physics of the Higgs boson.

\section{ACKNOWLEDGMENTS}

We are grateful to many people with whom we have discussed this analysis, including Gauthier Durieux, Christophe Grojean, Jiayin Gu, Howard Haber, Jenny List, Tomohisa Ogawa, Tomohiko Tanabe, Kechen Wang, Liantao Wang, and Jacqueline Yan. T. B., S. J., and M. E. P. were supported by the U.S. Department of Energy under contract No. DE-AC02-76SF00515. T. B. was also supported by a KEK Short-Term Invited Fellowship. He thanks the KEK ILC group for hospitality during this visit. S. J. was also supported by the National Research Foundation of Korea under Grants No. 2015R1A4A1042542 and No. 2017R1D1A1B03030820. K. F. was supported by the Japan Society for the Promotion of Science (JSPS) under Grants-in-Aid for Science Research 16H02173 and 16H02176. J. T. was supported by JSPS under Grant-inAid $15 \mathrm{H} 02083$.

\section{APPENDIX A: EXPANSIONS IN SMALL PARAMETERS USED IN OUR ANALYSIS}

In this appendix, we list the expansions in SM coupling shifts and $c_{I}$ operator coefficients used in the analysis of this paper. The notation is $\delta A=\Delta A / A$.

Observables depend on the underlying parameters both directly, through the coupling constants, and indirectly, through kinematic dependence on the masses $m_{W}, m_{Z}$, and $m_{h}$, which in turn depend on the coupling constants. In these formulas, we track both types of dependence. The variation of parameters contributing to the boson masses and the physical couplings is controlled by measurements of these masses and couplings that are included in our fit.

Expansions of boson field strength renormalizations:

$$
\begin{aligned}
\delta Z_{W} & =\left(8 c_{W W}\right), \\
\delta Z_{Z} & =c_{w}^{2}\left(8 c_{W W}\right)+2 s_{w}^{2}\left(8 c_{W B}\right)+s_{w}^{4} / c_{w}^{2}\left(8 c_{B B}\right), \\
\delta Z_{A} & =s_{w}^{2}\left(\left(8 c_{W W}\right)-2\left(8 c_{W B}\right)+\left(8 c_{B B}\right)\right), \\
\delta Z_{A Z} & =s_{w} c_{w}\left(\left(8 c_{W W}\right)-\left(1-\frac{s_{w}^{2}}{c_{w}^{2}}\right)\left(8 c_{W B}\right)-\frac{s_{w}^{2}}{c_{w}^{2}}\left(8 c_{B B}\right)\right), \\
\delta Z_{h} & =-c_{H} .
\end{aligned}
$$

Expansions of bare couplings:

$$
\begin{aligned}
\delta\left[g^{2}+g^{\prime 2}\right]^{1 / 2} & =c_{w}^{2} \delta g+s_{w}^{2} \delta g^{\prime}, \\
\delta\left(g g^{\prime} /\left[g^{2}+g^{\prime 2}\right]^{1 / 2}\right) & =s_{w}^{2} \delta g+c_{w}^{2} \delta g^{\prime}, \\
\delta s_{w} & =-c_{w}^{2}\left(\delta g-\delta g^{\prime}\right), \\
\delta c_{w} & =s_{w}^{2}\left(\delta g-\delta g^{\prime}\right) .
\end{aligned}
$$

Expansions of physical couplings:

$$
\begin{aligned}
\delta e= & s_{w}^{2} \delta g+c_{w}^{2} \delta g^{\prime}+\frac{1}{2} \delta Z_{A} \\
\delta g_{L}= & \frac{1}{\left(1 / 2-s_{w}^{2}\right)}\left[c_{w}^{2}\left(\frac{1}{2}+s_{w}^{2}\right) \delta g-s_{w}^{2}\left(\frac{1}{2}+c_{w}^{2}\right) \delta g^{\prime}+\frac{1}{2}\left(c_{H L}+c_{H L}^{\prime}\right)\right. \\
& \left.+\frac{1}{4} c_{w}^{2}\left(1+2 s_{w}^{2}\right)\left(8 c_{W W}\right)-\frac{1}{2} s_{w}^{2}\left(1-2 s_{w}^{2}\right)\left(8 c_{W B}\right)-\frac{1}{4} \frac{s_{w}^{4}}{c_{w}^{2}}\left(1+2 c_{w}^{2}\right)\left(8 c_{B B}\right)\right], \\
\delta g_{R}= & -c_{w}^{2} \delta g+\left(1+c_{w}^{2}\right) \delta g^{\prime}-\frac{1}{2 s_{w}^{2}} c_{H E}-\frac{1}{2} c_{w}^{2}\left(8 c_{W W}\right)+c_{w}^{2}\left(8 c_{W B}\right)+\frac{1}{2} \frac{s_{w}^{2}}{c_{w}^{2}}\left(1+c_{w}^{2}\right)\left(8 c_{B B}\right), \\
\delta g_{W}= & \delta g+c_{H L}^{\prime}+\frac{1}{2}\left(8 c_{W W}\right), \quad \delta g_{Z}=\left(1+s_{w}^{2}\right) \delta g-s_{w}^{2} \delta g^{\prime}+\frac{1}{2} \delta Z_{Z}+\frac{s_{w}}{c_{w}} \delta Z_{A Z} .
\end{aligned}
$$

Expansions of boson masses:

$$
\delta m_{W}=\delta g+\delta v+\frac{1}{2} \delta Z_{W}, \quad \delta m_{Z}=c_{w}^{2} \delta g+s_{w}^{2} \delta g^{\prime}+\delta v-\frac{1}{2} c_{T}+\frac{1}{2} \delta Z_{Z}, \quad \delta m_{h}=\frac{1}{2} \delta \bar{\lambda}+\delta v+\frac{1}{2} \delta Z_{h} .
$$


Expansions of precision electroweak observables:

$$
\begin{aligned}
\delta \alpha^{-1}= & -2 \delta e, \\
\delta G_{F}= & -2 \delta v+2 c_{H L}^{\prime}, \\
\delta A_{\ell}= & \frac{4 g_{L}^{2} g_{R}^{2}\left(\delta g_{L}-\delta g_{R}\right)}{\left(g_{L}^{2}+g_{R}^{2}\right)\left(g_{L}^{2}-g_{R}^{2}\right)} \\
\delta \Gamma_{\ell}= & \delta m_{Z}+\frac{2 g_{L}^{2} \delta g_{L}+2 g_{R}^{2} \delta g_{R}}{\left(g_{L}^{2}+g_{R}^{2}\right)}, \\
\delta \Gamma_{W, \ell}= & \delta m_{W}+2 \delta g_{W}, \\
\delta \Gamma_{W}= & 2 \delta g+\delta m_{W}+\delta Z_{W}+2 C_{W}, \\
\delta \Gamma_{Z}= & 2 c_{w}^{2}\left(1+2 \mathcal{Q} s_{w}^{2}\right) \delta g+2 s_{w}^{2}\left(1-2 \mathcal{Q} c_{w}^{2}\right) \delta g^{\prime} \\
& +\delta m_{Z}+\delta Z_{Z}+\mathcal{Q} s_{w} c_{w} \delta Z_{Z A}+2 C_{Z},
\end{aligned}
$$

where, in the last line $\mathcal{Q}=0.529$.
Expansions of Higgs coupling parameters:

$$
\begin{aligned}
\eta_{W} & =-\frac{1}{2} c_{H}+2 \delta m_{W}-\delta v, \\
\eta_{Z} & =-\frac{1}{2} c_{H}+2 \delta m_{Z}-\delta v-c_{T}, \\
\eta_{Z Z} & =-c_{H}+2 \delta m_{Z}-2 \delta v-5 c_{T}, \\
\eta_{h} & =-\frac{3}{2} c_{H}+c_{6}+\delta \bar{\lambda}+\delta v .
\end{aligned}
$$

Expansions of effective $W$ vertex parameters:

$$
\begin{aligned}
& \delta g_{Z, \mathrm{eff}}=\delta g_{Z}+\frac{1}{c_{w}^{2}}\left(\left(c_{w}^{2}-s_{w}^{2}\right) \delta g_{L}+s_{w}^{2} \delta g_{R}-2 \delta g_{W}\right), \\
& \delta \kappa_{A, \mathrm{eff}}=\left(c_{w}^{2}-s_{w}^{2}\right)\left(\delta g_{L}-\delta g_{R}\right)+2\left(\delta e-\delta g_{W}\right)+\left(8 c_{W B}\right), \\
& \delta \lambda_{A, \mathrm{eff}}=-6 g^{2} c_{3 W} .
\end{aligned}
$$

Expansions of $e^{+} e^{-} \rightarrow Z h$ parameters:

$$
\begin{aligned}
& a_{L}=\eta_{Z}+\delta g_{L}+\frac{\left(s-m_{Z}^{2}\right)}{2 m_{Z}^{2}} \frac{\left(c_{H L}+c_{H L}^{\prime}\right)}{\left(1 / 2-s_{w}^{2}\right)}+k_{Z} \delta m_{Z}+k_{h} \delta m_{h}, \\
& a_{R}=\eta_{Z}+\delta g_{R}-\frac{\left(s-m_{Z}^{2}\right)}{2 m_{Z}^{2}} \frac{c_{H E}}{s_{w}^{2}}+k_{Z} \delta m_{Z}+k_{h} \delta m_{h}, \\
& b_{L}=\frac{1}{\left(1-2 s_{w}^{2}\right)}\left\{c_{w}^{2}\left(1-2 s_{w}^{2} \frac{m_{Z}^{2}}{s}\right)\left(8 c_{W W}\right)+2 s_{w}^{2}\left(1-2 s_{w}^{2}\right) \frac{m_{Z}^{2}}{s}\left(8 c_{W B}\right)-\frac{1}{c_{w}^{2}}\left(1-2 c_{w}^{2} \frac{m_{Z}^{2}}{s}\right)\left(8 c_{B B}\right)\right\}, \\
& b_{R}=c_{w}^{2} \frac{m_{Z}^{2}}{s}\left(8 c_{W W}\right)+\left(1-\left(1-2 s_{w}^{2}\right) \frac{m_{Z}^{2}}{s}\right)\left(8 c_{W B}\right)+\frac{s_{w}^{2}}{c_{w}^{2}}\left(1-c_{w}^{2} \frac{m_{Z}^{2}}{s}\right)\left(8 c_{B B}\right) .
\end{aligned}
$$

In the formulas for $a_{L}$ and $a_{R}$,

$$
\begin{aligned}
k_{Z} & =\frac{2 m_{Z}^{2}}{s-m_{Z}^{2}}+\frac{E_{Z} m_{Z}^{2}}{2 k^{2} \sqrt{s}}-\frac{m_{Z}^{2}}{2 k^{2}}-\frac{E_{Z}^{2} / m_{Z}^{2}}{\left(2+E_{Z}^{2} / m_{Z}^{2}\right)}\left(1-\frac{m_{Z}^{2}}{E_{Z} \sqrt{s}}\right), \\
k_{h} & =-\frac{E_{Z} m_{h}^{2}}{2 k^{2} \sqrt{s}}-\frac{E_{Z}^{2} / m_{Z}^{2}}{\left(2+E_{Z}^{2} / m_{Z}^{2}\right)} \frac{m_{h}^{2}}{E_{Z} \sqrt{s}} .
\end{aligned}
$$

Expansions of $\sigma\left(e^{+} e^{-} \rightarrow \nu \bar{\nu} h\right)$ for different c.m. energies:

$$
\begin{aligned}
& \delta \sigma(250)=2 \eta_{W}-2 \delta v+2 \delta g_{W}-1.6 \delta m_{W}-3.7 \delta m_{h}-0.22 \delta Z_{W}-6.4 c_{H L}^{\prime}-0.37\left(c_{H L}-c_{H L}^{\prime}\right), \\
& \delta \sigma(350)=2 \eta_{W}-2 \delta v+2 \delta g_{W}-1.2 \delta m_{W}-2.0 \delta m_{h}-0.32 \delta Z_{W}-7.5 c_{H L}^{\prime}-0.28\left(c_{H L}-c_{H L}^{\prime}\right), \\
& \delta \sigma(380)=2 \eta_{W}-2 \delta v+2 \delta g_{W}-1.1 \delta m_{W}-1.7 \delta m_{h}-0.34 \delta Z_{W}-7.8 c_{H L}^{\prime}-0.26\left(c_{H L}-c_{H L}^{\prime}\right), \\
& \delta \sigma(500)=2 \eta_{W}-2 \delta v+2 \delta g_{W}-0.85 \delta m_{W}-1.2 \delta m_{h}-0.39 \delta Z_{W}-8.8 c_{H L}^{\prime}-0.19\left(c_{H L}-c_{H L}^{\prime}\right) .
\end{aligned}
$$


Expansions of Higgs boson partial widths:

$$
\begin{aligned}
\delta \Gamma(h \rightarrow b \bar{b}) & =-c_{H}+2 c_{b \Phi}, \\
\delta \Gamma(h \rightarrow c \bar{c}) & =-c_{H}+2 c_{c \Phi}, \\
\delta \Gamma\left(h \rightarrow \tau^{+} \tau^{-}\right) & =-c_{H}+2 c_{\tau \Phi}, \\
\delta \Gamma\left(h \rightarrow \mu^{+} \mu^{-}\right) & =-c_{H}+2 c_{\mu \Phi}, \\
\delta \Gamma(h \rightarrow g g) & =-c_{H}+2 c_{g \Phi}, \\
\delta \Gamma\left(h \rightarrow W W^{*}\right) & =2 \eta_{W}-2 \delta v-11.7 \delta m_{W}+13.6 \delta m_{h}-0.75 \delta Z_{W}-0.88 C_{W}+1.06 \delta \Gamma_{W}, \\
\delta \Gamma\left(h \rightarrow Z Z^{*}\right) & =2 \eta_{Z}-2 \delta v-13.8 \delta m_{Z}+15.6 \delta m_{h}-0.50 \delta Z_{Z}-1.02 C_{Z}+1.18 \delta \Gamma_{Z}, \\
\delta \Gamma(h \rightarrow \gamma \gamma) & =528 \delta Z_{A}-c_{H}+4 \delta e+4.2 \delta m_{h}-1.3 \delta m_{W}-2 \delta v, \\
\delta \Gamma(h \rightarrow Z \gamma) & =290 \delta Z_{A Z}-c_{H}-2\left(1-3 s_{W}^{2}\right) \delta g+6 c_{w}^{2} \delta g^{\prime}+\delta Z_{A}+\delta Z_{Z}+9.6 \delta m_{h}-6.5 \delta m_{Z}-2 \delta v .
\end{aligned}
$$

Expansions of $\sigma\left(e^{+} e^{-} \rightarrow Z h h\right)$ at $\sqrt{s}=500 \mathrm{GeV}$ for states of given $e^{+} e^{-}$beam polarization:

$$
\begin{aligned}
\delta \sigma(L)= & 2 \delta g_{L}+1.40 \eta_{Z}+1.02 \eta_{Z Z}+18.6 \delta Z_{Z}+24.8 \delta Z_{A Z}+0.56 \eta_{h}-1.58 c_{H}+108.3\left(c_{H L}+c_{H L}^{\prime}\right)-3.9 \delta m_{h}+3.5 \delta m_{Z}, \\
\delta \sigma(R)= & 2 \delta g_{R}+1.40 \eta_{Z}+1.02 \eta_{Z Z}+18.6 \delta Z_{Z}-28.7 \delta Z_{A Z}+0.56 \eta_{h}-1.58 c_{H}-125.5 c_{H E}-3.9 \delta m_{h}+3.5 \delta m_{Z}, \\
\delta \sigma(U)= & 1.15 \delta g_{L}+0.85 \delta g_{R}+1.40 \eta_{Z}+1.02 \eta_{Z Z}+18.6 \delta Z_{Z}+2.0 \delta Z_{A Z}+0.56 \eta_{h}-1.58 c_{H}+62.1\left(c_{H L}+c_{H L}^{\prime}\right) \\
& -53.5 c_{H E}-3.9 \delta m_{h}+3.5 \delta m_{Z} .
\end{aligned}
$$

In these equations $L$ refers to the beam polarization state $e_{L}^{-} e_{R}^{+}, R$ refers to the beam polarization state $e_{L}^{-} e_{R}^{+}$, and $U$ refers to unpolarized beams. To find the expressions for arbitrary polarizations, it is useful to have the total cross sections for the two completely polarized beam configurations: $\sigma(L)=0.36 \mathrm{fb}, \sigma(R)=0.27 \mathrm{fb}$.

\section{APPENDIX B: VALUES FOR PROJECTED UNCERTAINTIES INPUT INTO OUR ANALYSIS}

The 13-parameter fit described in Sec. V used as inputs projected uncertainties in precision electroweak observables, LHC measurements of ratios of Higgs boson branching ratios, and measurements of the $a$ and $b$ parameters of $e^{+} e^{-} \rightarrow Z h$ at the $500 \mathrm{GeV}$ ILC. For the precision electroweak inputs, we have taken the values listed in Table I, including the future improvements quoted there. For LHC measurements, we have used as our inputs

$$
\begin{aligned}
\delta\left(\mathrm{BR}\left(h \rightarrow Z Z^{*}\right) / \mathrm{BR}(h \rightarrow \gamma \gamma)\right) & =2 \%, \\
\delta(\operatorname{BR}(h \rightarrow Z \gamma) / \mathrm{BR}(h \rightarrow \gamma \gamma)) & =31 \%, \\
\delta\left(\operatorname{BR}\left(h \rightarrow \mu^{+} \mu^{-}\right) / \mathrm{BR}(h \rightarrow \gamma \gamma)\right) & =12 \%
\end{aligned}
$$

as described in Sec. III. For the $a$ and $b$ parameter measurements, we have used the estimates [54]

\begin{tabular}{llll} 
beam polarization & $\delta a$ & $\delta b$ & $\rho(a, b)$ \\
\hline$-80 \% /+30 \%$ & 4.0 & 0.70 & 84.8 \\
$+80 \% /-30 \%$ & 4.2 & 0.75 & 86.5
\end{tabular}

with all numbers in $\%$.

The final fit described in Sec. VIII, which uses 22 parameters, makes use of a much larger number of inputs. These are listed in the Appendix of Ref. [23]. The full set of linear relations given in Appendix A, and the final $22 \times 22$ covariance matrices for the fit parameters given by the ILC 250 fit and the full ILC fit are given in the files CandV250.txt and CandV500.txt provided in the Supplemental Material of Ref. [23].

\section{APPENDIX C: RELATION BETWEEN THE EFT AND $S, T$ FORMALISMS}

In the $S, T$ formalism for the interpretation of precision electroweak measurements [37], we define a reference value of the weak mixing angle from the quantities $\alpha\left(m_{Z}^{2}\right), m_{Z}$, and $G_{F}$ and then compare the predictions for other precision electroweak observables to expectations based on this value. More specifically, we define $\sin ^{2} \theta_{0}$ by

$$
4 s_{0}^{2} c_{0}^{2}=\frac{4 \pi \alpha}{\sqrt{2} G_{F} m_{Z}^{2}} .
$$


Then we can write expressions for precision electroweak observables in terms of $s_{0}^{2}$. The variations of the SM parameters conveniently cancel out of these formulas at leading order. For example,

$$
\begin{aligned}
m_{W}^{2} / m_{Z}^{2}= & c_{0}^{2}+\frac{c_{0}^{2}}{c_{0}^{2}-s_{0}^{2}}\left(c_{0}^{2} c_{T}-2 s_{0}^{2}\left(c_{H L}^{\prime}+\left(8 c_{W B}\right)\right)\right. \\
s_{*}^{2}= & s_{0}^{2}+\frac{s_{0}^{2}}{c_{0}^{2}-s_{0}^{2}}\left(c_{H L}^{\prime}+\left(8 c_{W B}\right)-c_{0}^{2} c_{T}\right) \\
& -\frac{1}{2} c_{H E}-s_{0}^{2}\left(c_{H L}-c_{H E}\right),
\end{aligned}
$$

where $s_{*}^{2}$ is the value of the weak mixing angle that governs the polarization asymmetries at the $Z$ pole.

The $S$ and $T$ parameters are defined so that, in the approximation in which all precision electroweak corrections arise from vacuum polarization diagrams, the formulas $(\mathrm{C} 2)$ take the form

$$
\begin{aligned}
m_{W}^{2} / m_{Z}^{2} & =c_{0}^{2}+\frac{\alpha c_{0}^{2}}{c_{0}^{2}-s_{0}^{2}}\left(-\frac{1}{2} S+c_{0}^{2} T\right), \\
s_{*}^{2} & =s_{0}^{2}+\frac{\alpha}{c_{0}^{2}-s_{0}^{2}}\left(\frac{1}{4} S-s_{0}^{2} c_{0}^{2} T\right) .
\end{aligned}
$$

Then we can identify

$$
\alpha S=4 s_{0}^{2}\left(8 c_{W B}+c_{H L}^{\prime}\right), \quad \alpha T=c_{T}
$$

The $S, T$ formalism was quite appropriate for the experimental situation of the early 1990s, when $\alpha, G_{F}$, and $m_{Z}$ were by far the best-measured electroweak parameters. Today, the uncertainties in $m_{W}$ and $A_{\ell}$ have improved to the point where these observables should be treated on the same footing. The formalism used in this paper is more democratic with respect to possible choices of the reference electroweak parameters.
[1] G. Aad et al. (ATLAS Collaboration), Phys. Lett. B 716, 1 (2012).

[2] S. Chatrchyan et al. (CMS Collaboration), Phys. Lett. B 716, 30 (2012).

[3] G. F. Giudice, C. Grojean, A. Pomarol, and R. Rattazzi, J. High Energy Phys. 06 (2007) 045.

[4] R. Contino, M. Ghezzi, C. Grojean, M. Muhlleitner, and M. Spira, J. High Energy Phys. 07 (2013) 035.

[5] N. Craig, M. Farina, M. McCullough, and M. Perelstein, J. High Energy Phys. 03 (2015) 146.

[6] D. de Florian et al. (LHC Higgs Cross Section Working Group), arXiv:1610.07922.

[7] Some analyses that approach this goal are given in A. Pomarol and F. Riva, J. High Energy Phys. 01 (2014) 151; R. S. Gupta, A. Pomarol, and F. Riva, Phys. Rev. D 91, 035001 (2015); J. de Blas, M. Ciuchini, E. Franco, S. Mishima, M. Pierini, L. Reina, and L. Silvestrini, J. High Energy Phys. 12 (2016) 135.

[8] D. E. Morrissey and M. J. Ramsey-Musolf, New J. Phys. 14, 125003 (2012).

[9] ATLAS Collaboration, Report No. ATL-PHYS-PUB-2017001, 2017.

[10] CMS Collaboration, Report No. CMS PAS FTR-16-002, 2017.

[11] R. Contino et al., CERN Yellow Report 3, 255 (2017).

[12] J. Tian and K. Fujii, Nucl. Part. Phys. Proc. 273-275, 826 (2016).

[13] H. Abramowicz et al., Eur. Phys. J. C 77, 475 (2017).

[14] M. McCullough, Phys. Rev. D 90, 015001 (2014); 92, 039903(E) (2015).

[15] R. Contino, C. Grojean, D. Pappadopulo, R. Rattazzi, and A. Thamm, J. High Energy Phys. 02 (2014) 006.
[16] F. Goertz, A. Papaefstathiou, L. L. Yang, and J. Zurita, J. High Energy Phys. 04 (2015) 167.

[17] A. Azatov, R. Contino, G. Panico, and M. Son, Phys. Rev. D 92, 035001 (2015).

[18] A. Carvalho et al., arXiv:1608.06578.

[19] S. Di Vita, C. Grojean, G. Panico, M. Riembau, and T. Vantalon, J. High Energy Phys. 09 (2017) 069.

[20] J. Cohen, S. Bar-Shalom, and G. Eilam, Phys. Rev. D 94, 035030 (2016).

[21] A. Falkowski and F. Riva, J. High Energy Phys. 02 (2015) 039.

[22] A. Falkowski, M. Gonzalez-Alonso, A. Greljo, D. Marzocca, and M. Son, J. High Energy Phys. 02 (2017) 115.

[23] T. Barklow et al., preceding article, Phys. Rev. D 97, 053003 (2018).

[24] D. M. Asner et al., in Planning the Future of U.S. Particle Physics The Snowmass 2013 Proceedings, edited by N. Graf, J.L. Rosner, and M.E. Peskin, http://www.slac .stanford.edu/econf/C1307292/, arXiv:1310.0763.

[25] K. Fujii et al. (LCC Physics Working Group), arXiv:1506 .05992 .

[26] T. Barklow et al. (LCC Parameters Working Group), arXiv: 1506.07830 .

[27] B. Grzadkowski, M. Iskrzynski, M. Misiak, and J. Rosiek, J. High Energy Phys. 10 (2010) 085.

[28] B. Henning, X. Lu, T. Melia, and H. Murayama, J. High Energy Phys. 08 (2017) 016.

[29] J. D. Wells and Z. Zhang, J. High Energy Phys. 01 (2016) 123.

[30] A. Falkowski, B. Fuks, K. Mawatari, K. Mimasu, F. Riva, and V. Sanz, Eur. Phys. J. C 75, 583 (2015).

[31] B. Henning, X. Lu, and H. Murayama, J. High Energy Phys. 01 (2016) 023. 
[32] I. Brivio and M. Trott, arXiv:1706.08945.

[33] A. Alloul, B. Fuks, and V. Sanz, J. High Energy Phys. 04 (2014) 110.

[34] C.F. Dürig, Measuring the Higgs self-coupling at the International Linear Collider, Report No. DESY-THESIS2016-027, 2016.

[35] K. Endo and Y. Sumino, J. High Energy Phys. 05 (2015) 030.

[36] K. Hashino, S. Kanemura, and Y. Orikasa, Phys. Lett. B 752, 217 (2016).

[37] M. E. Peskin and T. Takeuchi, Phys. Rev. Lett. 65, 964 (1990); Phys. Rev. D 46, 381 (1992).

[38] C. Patrignani et al., Chin. Phys. C 40, 100001 (2016).

[39] H. Baer et al., arXiv:1306.6352, https://www.linearcollider .org/ILC/Publications/Technical-Design-Report.

[40] V. Barger, T. Han, P. Langacker, B. McElrath, and P. Zerwas, Phys. Rev. D 67, 115001 (2003).

[41] N. Craig, C. Englert, and M. McCullough, Phys. Rev. Lett. 111, 121803 (2013).

[42] J. Erler and A. Freitas, in Ref. [38].

[43] S. Schael et al., Phys. Rep. 427, 257 (2006).

[44] A. Kotwal et al., in Planning the Future of U.S. Particle Physics The Snowmass 2013 Proceedings, edited by N. Graf, J. L. Rosner, and M.E. Peskin, http://www.slac .stanford.edu/econf/C1307292/, arXiv:1310.6708.

[45] J. Yan, S. Watanuki, K. Fujii, A. Ishikawa, D. Jeans, J. Strube, J. Tian, and H. Yamamoto, Phys. Rev. D 94, 113002 (2016).
[46] R. Hawkings and K. Mönig, Eur. Phys. J. direct 1, 1 (2000).

[47] K. Hagiwara, R. D. Peccei, D. Zeppenfeld, and K. Hikasa, Nucl. Phys. B282, 253 (1987).

[48] G. Gounaris et al., in Workshop on Physics at LEP2, edited by G. Altarelli, T. Sjöstrand, and F. Zwirner (CERN, Geneva, 1996).

[49] I. Marchesini, Report No. DESY-THESIS-2011-044.

[50] A. Rosca, Nucl. Part. Phys. Proc. 273-275, 2226 (2016).

[51] We ignore the logically possible but highly fine-tuned hypothesis that the new physics contribution to these decays is almost exactly $(-2)$ times the SM contribution.

[52] ATLAS Collaboration, Report No. ATL-PHYS-PUB-2014016, 2014.

[53] ATLAS Collaboration, Report No. ATL-PHYS-PUB-2014006, 2014.

[54] T. Ogawa, in Proceedings of the EPS Conference on High Energy Physics 2017, Venice, 5-12 July, 2017 (to be published), https://indico.cern.ch/event/466934/contributions/ 2588482/.

[55] C. Grojean, E. Salvioni, M. Schlaffer, and A. Weiler, J. High Energy Phys. 05 (2014) 022.

[56] M. Grazzini, A. Ilnicka, M. Spira, and M. Wiesemann, J. High Energy Phys. 03 (2017) 115. 